\title{
Chiral anomalies induced by gravitational waves
}

\author{
Adrian del Rio ${ }^{1}$ \\ ${ }^{1}$ Institute for Gravitation and the Cosmos, Physics Department, \\ Penn State, University Park, PA 16802-6300, USA.
}

(Dated: June 17, 2021)

\begin{abstract}
Chiral symmetries in field theory are typically affected by an anomaly in the quantum theory. This anomaly emerges when one introduces an interaction with a Yang-Mills or gravitational background. Physical applications of this quantum effect have been traditionally connected to topological questions of the background field and the study of instantons. We show here how one can alternatively find situations of physical interest that only involve ordinary, but dynamical solutions of the background field equations. More precisely, we show that solutions to the Einstein (Maxwell) equations are able to trigger the chiral anomaly if and only if they admit a flux of gravitational (electromagnetic) radiation with net circular polarization. As a consequence, astrophysical systems that admit such radiation spontaneously generate a flux of particles with net helicity from the quantum vacuum.
\end{abstract}

\section{INTRODUCTION}

It is well-known that strong gravitational fields can affect the vacuum fluctuations of quantum fields and lead to important physical phenomena. From the pioneer work of Parker on particle creation in expanding universes [1], to the subsequent discover by Hawking of thermal emission during a gravitational collapse [2], different phenomena of quantum origin can arise if a quantum field propagates on a dynamical, gravitational background. One of these quantum effects is related to the emergence of anomalies due to spacetime curvature.

An anomaly is understood as the failure of some Noether symmetry of a classical field theory to persist after the quantization. More precisely, when the classical conservation law of a Noether current breaks down in the quantum theory, the associated symmetry is said to be anomalous [3]. Their discovery was initiated in the late 60's with the fermion axial or chiral anomaly [4, 5], motivated with the aim of understanding the observed phenomenon of the pion decay into two photons. Since then, the field has grown enormously, leading to the discovery of many more anomalous symmetries of diverse nature (conformal, gauge, etc), and to a rich interplay with differential geometry and topology [6]. From a physical viewpoint, anomalies were found useful to address key conceptual questions in the standard model of particles (U(1) problem, strong CP violation in QCD [7]) and cosmology (baryogenesis). Our goal in this paper is to point out and develop an unexplored aspect of chiral anomalies that, remarkably, turns out to have a simple physical interpretation, and could lead to new physical applications in gravity and electrodynamics.

For definiteness, let $\psi(x)$ be a Dirac field interacting with a classical Yang-Mills background of field strength $F_{a b}$ in Minkowski spacetime $\left(\mathbb{R}^{4}, \eta_{a b}\right)$, with coupling constant $g$. Let $\gamma^{a}$ be the Dirac matrices, and let $\gamma^{5}:=i \gamma^{0} \gamma^{1} \gamma^{2} \gamma^{3}$ be the chiral matrix. In the massless limit, the standard action of this theory possesses a (global) Noether symmetry generated by the transformation $\psi(x) \rightarrow e^{i \gamma^{5} \theta} \psi(x), \theta \in \mathbb{R}$, that leads to a Noether current: $j_{5}^{a}(x)=\bar{\psi}(x) \gamma^{a} \gamma^{5} \psi(x)$. This is the well-known (abelian) chiral symmetry. This current is conserved for solutions $\psi(x)$ of the Dirac equation of motion, $\nabla_{a} j_{5}^{a}(x) \approx 0$. In the quantum theory, however, off-shell contributions yield

$$
\left\langle\nabla_{a} j_{5}^{a}\right\rangle=\frac{-g^{2}}{16 \pi^{2}} \operatorname{Tr} F_{a b}{ }^{*} F^{a b} \neq 0
$$

thus spoiling the classical conservation law. This is the indication that the classical symmetry is anomalous in the quantum theory. Denoting by $\psi_{L}=1 / 2\left(\mathbb{I}+\gamma^{5}\right) \Psi$ and $\psi_{R}=1 / 2\left(\mathbb{I}-\gamma^{5}\right) \Psi$ the right-handed and left-handed chiral sectors of the Dirac field, respectively, the associated Noether charge can be written as $Q_{5}(t)=\int_{\Sigma} d^{3} x \sqrt{h}\left(\psi_{R}^{\dagger} \psi_{R}-\right.$ $\left.\psi_{L}^{\dagger} \psi_{L}\right)$, which is a measure of the net difference between right-handed (positive-helicity particles plus negativehelicity antiparticles) and left-handed fermions (hegative-helicity particles plus positive-helicity antiparticles). While this difference is preserved by the equation of motion for classical fields, $\dot{Q}_{5}(t) \approx 0$, the emergence of the anomaly indicates that quantum fluctuations are able to induce a change in time given by

$$
\left\langle\hat{Q}_{5}\left(t_{2}\right)\right\rangle-\left\langle\hat{Q}_{5}\left(t_{1}\right)\right\rangle=\int_{\left[t_{1}, t_{2}\right] \times \Sigma} d^{4} x \sqrt{-\eta}\left\langle\nabla_{a} j_{5}^{a}\right\rangle=\frac{-g^{2}}{16 \pi^{2}} \int_{\left[t_{1}, t_{2}\right] \times \Sigma} d^{4} x \sqrt{-\eta} \operatorname{Tr} F_{a b}{ }^{*} F^{a b},
$$

if and only if the RHS of this equation is non-vanishing. What does the anomalous time dependence of the chiral charge imply physically? Using S-matrix theory and Bogoliubov transformations, it can be explicitly shown 8] that the Yang-Mills field creates and destroys fermions in such a way that $\left\langle Q_{5}\left(t_{2}\right)\right\rangle-\left\langle Q_{5}\left(t_{1}\right)\right\rangle$, which is a measure of 
asymmetric particle creation, is given precisely by the amount predicted by the RHS of the previous equation. Thus, the anomalous temporal evolution of the chiral charge is physically interpreted as a phenomenon of asymmetric particle creation by a dynamical background: a non-trivial gauge field is able to excite spontaneously a net number of right-handed fermions over left-handed ones from the quantum vacuum, or viceversa.

In a similar fashion, it has been recently shown $9-12$ that the analogous chiral symmetry in electrodynamics, most popularly known as electric-magnetic duality symmetry of source-free Maxwell equations, suffers from a similar anomaly when a non-trivial spacetime background $\left(\mathbb{R}^{4}, g_{a b}\right)$ is introduced. More precisely, under a chiral rotation ${ }^{ \pm} F(x) \rightarrow e^{\mp i \theta}{ }^{ \pm} F(x)$ of the self-dual and anti self-dual sectors of the electromagnetic field, ${ }^{ \pm} F_{a b}=\frac{1}{2}\left[F_{a b} \pm i^{*} F_{a b}\right]$, the usual action for the source-free Maxwell theory remains invariant. This leads to a Noether current that, for solutions of the field equations, reads $J_{5}^{a} \approx A_{b}{ }^{*} F^{a b}-Z_{b} F^{a b}$. Although classically conserved, it was found in [9-12] that quantum fluctuations of the electromagnetic field produce

$$
\left\langle\nabla_{a} J_{5}^{a}\right\rangle=\frac{-1}{96 \pi^{2}} R_{a b c d}{ }^{*} R^{a b c d}
$$

if spacetime curvature $R_{a b c}{ }^{d}$ is considered. The associated Noether charge can be expressed as the difference between right-handed and left-handed photons, and, while Maxwell equations guarantee that $\dot{Q}_{5} \approx 0$ for the classical function $Q_{5}(t)$, off-shell contributions can spontaneously make this quantity change in time if and only if the RHS of

$$
\left\langle\hat{Q}_{5}\left(t_{2}\right)\right\rangle-\left\langle\hat{Q}_{5}\left(t_{1}\right)\right\rangle=\frac{-1}{96 \pi^{2}} \int_{\left[t_{1}, t_{2}\right] \times \Sigma} d^{4} x \sqrt{-g} R_{a b c d}{ }^{*} R^{a b c d},
$$

is different from zero. Again, the physical picture is analogous to the fermion case: a non-trivial gravitational background would be responsible to create spontaneosly a difference in the number of right- and left-handed circularly polarized photons from the quantum vacuum.

In general, the physical interpretation of chiral anomalies is strongly associated to the phenomenon of "levelcrossing". The Hamiltonian of the quantum field, which determines the energy of field modes, depends on the background field. Then, a non-trivial temporal evolution of the latter can make a positive-chirality mode with initial negative energy transform into a positive-chirality mode with final positive energy [8, 13]. In other words, the dynamical evolution of the Yang-Mills or gravitational field can reverse the helicity of field modes, producing as a result a net creation of helicity from the quantum vacuum: more particles of one helicity are created than particles of the opposite helicity.

The question we want to answer here is: what are the physical spacetime backgrounds that can induce this levelcrossing in the helicity of field modes? How is this dynamical evolution supposed to be? In the Yang-Mills case an important historical role has been played by instantons [14, 15]. Instantons are classical solutions to the Euclidean field equations of a non-abelian gauge theory which exhibit a non-trivial topology in the manifold of field configurations. Physically, they are interpreted as amplitudes that quantify quantum-mechanical transitions between topologicallyinequivalent vacua in the Hilbert space of gauge fields [14, 16 18]. Their use played a fundamental role in the 70-80 in addressing several problems of the standard model of particles and QCD, most notably the $U(1)$ problem [19]. But what about the gravitational case? Analogous solutions of the Euclidean Einstein's equations are also known for a long time 20, 21] and were baptised as gravitational instantons. While mathematically these solutions have a rich structure [22], their physical interpretation could be regarded as exotic, since it relies on quantum-gravity issues [23]. If one is only interested in studying applications of chiral anomalies, it can be more natural to look instead for more realistic spacetimes, i.e. for ordinary, Lorentzian solutions of Einsteins equations directly.

To our knowledge, this problem has not been proposed in the literature before. Since it is known that the integrand in (4) is locally a total derivative, a priori one expects that only non-trivial spacetime topologies can produce nonvanishing contributions, thereby the historical interest in instantons. However, in gravity this is not quite true because, at least for asymptotically flat spacetimes, the boundary of the spacetime, called null infinity [24], is non-trivial and can provide a contribution by means of the flux of gravitational waves (GWs) that enters/exits the spacetime. The goal of this paper is to fill this gap, and to open a new window for applications of chiral anomalies that go beyond the realm of topology or instanton calculus.

Our main result will be to show that chiral anomalies are intimately related to the circular polarization state of ordinary gravitational radiation in the spacetime background. More precisely, we shall prove that:

$$
\left\langle\hat{Q}_{5}(\text { scri }+)\right\rangle-\left\langle\hat{Q}_{5}(\text { scri- })\right\rangle=\int_{0}^{\infty} \frac{d \omega \omega^{3}}{24 \pi^{3}} \sum_{\ell m}\left[\left|h_{+}^{\ell m}(\omega)-i h_{\times}^{\ell m}(\omega)\right|^{2}-\left|h_{+}^{\ell m}(\omega)+i h_{\times}^{\ell m}(\omega)\right|^{2}\right],
$$

where $h_{+}, h_{\times}$denote the two GW linear polarization modes that reach future null infinity, emitted by an isolated gravitational source that is stationary at both past and future timelike infinities, but otherwise arbitrary. What this formula is indicating is that the more right(left)-handed gravitational radiation is emitted by a system, the more 
right(left)-handed particles will be excited from the quantum vacuum through the mechanism that produces the chiral anomaly. This is a realistic gravitational setting, with a clear and unambiguous physical meaning.

That an asymptotically flat spacetime background must emit gravitational waves in order to induce the quantum anomaly is an indication that only dynamical solutions of Einstein's equations are relevant in this question. 11 This should come with no surprise in light of the previous physical interpretation of anomalies in terms of asymmetric particle creation, since only dynamical gravitational fields are able to spontaneously create particles (and hence helicity) from the quantum vacuum. On the other hand, because the study dynamical solutions of Einstein's equations is a rather involved issue that typically requires numerical techniques, this could explain why only instantonic solutions (which are known in closed form) have only been considered so far in the study of chiral anomalies.

In a given sense the result that we obtain shares some parallelisms and interpretations with instantons. Namely, our result can be understood as tunneling between degenerate vacua of the asymptotically flat spacetime (associated to the degeneracy of gravitational connections at future null infinity [25, 26]), but in this case these transitions are produced classically simply by a flux of gravitational waves crossing null infinity (i.e. not through the usual quantummechanical tunneling). However, important conceptual differences exist. For instance, our result indicates that any spacetime that its manifold is homeomorphic to $\mathbb{R}^{4}$, but such that its metric is deformed with respect to Minkowski so as to allow the presence of circularly polarized gravitational radiation (i.e. to allow for curvature, in a specific form) will be able to induce the quantum anomaly and hence level-crossing of modes. Thus, our result has nothing to do with topological or global questions, but rather to the geometry of the spacetime. Moreover, it seems unlikely that these results could be simply obtained by a wick rotation of an instanton solution, since the former are purely dynamical (gravitational waves), and hence it does not look that they could be recovered from "static" euclidean solutions by any analytical continuation.

In light of the systematic detections of gravitational waves by the interferometers LIGO-Virgo in the last years [28], and given that gravitational backgrounds emitting these waves are intimately related to chiral anomalies, it is important to discuss astrophysical settings where these quantum effects could play a role in the underlying physics. What is the possible phenomenology that one could predict? This will be studied in detail in a separate paper. The present paper is an extended and a detailed discussion of the theoretical results presented already in [29].

We shall work with 4-dimensional spacetimes and the Levi-Civita connection. We follow Wald's [30] sign conventions. Namely, the metric signature is $(-,+,+,+)$, the Riemann tensor is defined by $\left[\nabla_{a}, \nabla_{b}\right] v_{c}=: R_{a b c}{ }^{d} v_{d}$ for any covector field $v_{d}$, the Ricci tensor is $R_{a b}:=R_{a c b}^{c}$, and the scalar curvature is $R:=g^{a b} R_{a b}$. Unless otherwise stated, all tensor fields will be considered smooth. In section II we use units in which $\hbar=c=1$, while in section III we use units in which $G=c=1$.

\section{A SIMPLE CASE: THE ELECTROMAGNETIC ANALOGUE}

Although we are ultimately interested in understanding the chiral anomaly induced by a gravitational background, the usual technical complications associated with the non-linearities of the gravitational field and Einstein's equations makes necessary working first with a simpler model. Consequently, let us focus in this section on the original AdlerBell-Jackiw chiral anomaly [4, 5], which is the electromagnetic analogue. The above complications are avoided due to the simplicity of Maxwell theory. More importantly, the final result will have such a simple physical interpretation that will guide us in the gravitational case.

\section{A. Setup and main calculation}

The Adler-Bell-Jackiw chiral anomaly is the anomalous non-conservation of the Noether current associated to the chiral symmetry of a Dirac spinor $\Psi(x)$. This anomaly arises when the spinor interacts with a background electromagnetic field $F_{a b}(x)$. The explicit expression for this anomaly can be read off from (10) if we identify the gauge group with $U(1)$ and $g^{2} \rightarrow e^{2}$. As emphasized in the introduction, the quantity of major physical interest is the Noether charge, whose failure to be preserved in time is determined by the integral over all spacetime $M$ :

$$
\left\langle\hat{Q}_{5}(\text { scri }+)\right\rangle-\left\langle\hat{Q}_{5}(\text { scri- })\right\rangle=-\frac{e^{2}}{8 \pi^{2}} \int_{M} F \wedge F=-\frac{e^{2}}{16 \pi^{2}} \int_{M} d^{4} x \sqrt{-\eta} F_{a b}{ }^{*} F^{a b} .
$$

\footnotetext{
${ }^{1}$ But not all dynamical spacetimes produce a non-vanishing Chern-Pontryagin. One can easily check that Fridmann-Lemaitre-RobertsonWalker metrics produce a zero result.

${ }^{2}$ In the context of the Atiyah-Patodi-Singer index theorem, this geometric contribution is essentially the one that must be subtracted from the Chern-Pontryagin integral in order to recover a topological quantity for manifolds with boundary, see [27]
} 
Our goal is to determine which class of electromagnetic backgrounds (i.e. solutions $F_{a b}$ of Maxwell equations) produce a non-vanishing chiral anomaly. To achieve this we need to analyze this integral and to study under which conditions it is not zero. We assume that this electromagnetic background $F_{a b}$ is produced by some electromagnetic sources $J^{a}$, that are smooth and with spatial compact support, but otherwise arbitrary.

First of all, it is not difficult to prove that for stationary solutions of Maxwell equations, $d F=0, d^{*} F={ }^{*} J$, eq. (6) above is identically zero. To see this, let us take a cartesian coordinate system $\{t, \vec{x}\}$. A stationary Maxwell field is a solution of Maxwell's equations that remains invariant under time translations, i.e. a solution that satisfies $\mathcal{L}_{k} F_{a b}=0$, where $\mathcal{L}_{k}$ denotes the Lie derivative along the generator of infinitesimal time-translations, $k=\partial / \partial t$. This condition is equivalent to $k^{a} F_{a b}=\nabla_{b} \Lambda$, where $\Lambda$ is a function, traditionally called the electrostatic potential. The integral of interest can be now rewritten as

$$
\begin{aligned}
\int_{M} d^{4} x \sqrt{-\eta} F_{a b}{ }^{*} F^{a b} & =-4 \int_{-\infty}^{\infty} d t \int_{\mathbb{R}^{3}} d^{3} \vec{x} k^{a} F_{a b} k_{c}{ }^{*} F^{c b}=-4 \int_{-\infty}^{\infty} d t \int_{\mathbb{R}^{3}} d^{3} \vec{x} \nabla_{b} \Lambda k_{c}{ }^{*} F^{c b} \\
& =-4 \int_{-\infty}^{\infty} d t \int_{\mathbb{R}^{3}} d^{3} \vec{x} \nabla_{b}\left(\Lambda k_{c}{ }^{*} F^{c b}\right),
\end{aligned}
$$

where in the first equality we used the $3+1$ decomposition of the metric, $\eta^{a b}=-k^{a} k^{b}+h^{a b}$, and ${ }^{*} F_{a b}=\frac{1}{2} \epsilon_{a b c d} F^{c d}$ to write $h^{a b} h^{c d} F_{a c}{ }^{*} F_{b d}=-2 k^{a} F_{a b} k_{c}{ }^{*} F^{c b}$; while in the third equality we used Maxwell equation $\nabla_{a}{ }^{*} F^{a b}=0$. Assuming standard fall-off conditions for the magnetic field and electrostatic potential at spatial infinity, $k^{a *} F_{a b} \nabla^{b} r \sim 1 / r^{3}$, $\Lambda \sim 1 / r$, the final result is zero.

We must look then for non-stationary solutions to Maxwell's equations. To guarantee convergence for the integral in time in eq. ((6) ), we shall assume that both at early and late times the solution of Maxwell equations approaches a stationary configuration. 3 During the non stationarity period, the dynamics of the electromagnetic sources will generate outgoing radiation propagating to infinity (we will assume no incoming electromagnetic radiation for simplicity). The study of outgoing radiation is most conveniently carried out within the framework of asymptotically Minwkoski spacetimes [31 33]. A detailed summary of this topic can be found in Appendix B, and we will provide the key points here. Let $\left(\mathbb{R}^{4}, \hat{\eta}_{a b}\right)$ denote our physical, Minkowski spacetime, and let $\left(M, \eta_{a b}\right)$ be the unphysical spacetime constructed from the physical one by a standard conformal compactification 4 The unphysical metric is related to the physical one by an ordinary conformal transformation: $\eta_{a b}=\Omega^{2}(x) \hat{\eta}_{a b}$. On the other hand, the unphysical manifold is just the physical one together with additional points attached smoothly to it: $M=\mathbb{R}^{4} \cup \mathcal{J}$. The set of all these new points constitute a null hypersurface $\mathcal{J}$, locally characterized by the condition $\Omega=0$, and with null normal $\eta^{a b} \nabla_{b} \Omega$. Physically, they represent the "points of (null) infinity", i.e. the points that can be asymptotically reached in the original spacetime by following outgoing, null geodesics.

The importance of this construction is that it allows to apply ordinary techniques in differential geometry to study the behaviour of fields in a neigbourhood of infinity (which now is just a boundary of the spacetime manifold). To do the calculation of interest, equation ([6), one further needs to carry the tensors of the original spacetime to the unphysical one. This is straightforward due to he invariance of the electromagnetic field under conformal transformations, $\hat{F}_{a b}=F_{a b}, \hat{A}_{a}=A_{a}$. Thus,

$$
-\frac{1}{2} \int_{\mathbb{R}^{4}} d^{4} x \sqrt{-\hat{\eta}} \hat{F}_{a b}{ }^{*} \hat{F}^{a b}=\int_{\mathbb{R}^{4}} \hat{F} \wedge \hat{F}=\int_{M} F \wedge F=-\frac{1}{2} \int_{M} d^{4} x \sqrt{-\eta} F_{a b}{ }^{*} F^{a b} .
$$

The key point now is to notice that, mathematically, $p_{1}(F)=-\frac{1}{8 \pi^{2}} F \wedge F$ is an invariant polynomial [6]. The Chern-Weil Theorem from the theory of characteristic classes (see Theorem 11.1 in [6] , for instance) states that the difference between two invariant polynomials, $p_{1}(F)-p_{1}\left(F^{\prime}\right)$, associated to two connection 1-forms, $A$ and $A^{\prime}$, is exact and determined by the transgression term $Q\left(A, A^{\prime}\right)[6,27]$ :

$$
p_{1}(F)-p_{1}\left(F^{\prime}\right)=d Q\left(A, A^{\prime}\right) .
$$

Because the spacetime $\left(\mathbb{R}^{4}, \hat{\eta}_{a b}\right)$ is trivial from the topological viewpoint, it admits a global flat connection $\hat{A}^{\prime}$. Due to conformal invariance and continuity, we have an electromagnetic potential that is pure gauge globally: $A^{\prime}=d \alpha$. Then, the difference $\theta=A-A^{\prime}$ represents the same physical electromagnetic potentia 5 . The transgression term can

${ }^{3}$ At early times the electromagnetic field is stationary for all $\vec{x} \in \mathbb{R}^{3}$ and using the same arguments as above we get $\int_{\mathbb{R}^{3}} d^{3} \vec{x} \nabla_{b}\left(\Lambda k_{c}{ }^{*} F^{c b}\right)=$ 0 . At late times $t$ the field is stationary only in a spacelike open region $U(t) \subset \mathbb{R}^{3}$ of radius $r(t)$ that does not intersect the electromagnetic waves generated during the intermediate non-stationary period. Because the waves propagate to future infinity, $r(t)=t+$ const, then $U(t \rightarrow \infty) \rightarrow \mathbb{R}^{3}$ and we find $\int_{U(t)} d^{3} \vec{x} \nabla_{b}\left(\Lambda k_{c}{ }^{*} F^{c b}\right)=\int d \mathbb{S}^{2} r(t)^{2} \Lambda(r(t)) k_{c}{ }^{*} F^{c b}(r(t)) \nabla_{b} r \sim t^{-2}$ as $t \rightarrow \infty$, which guarantees convergence of the integral.

${ }^{4}$ Because we shall be working with the unphysical spacetime all the time, we use the hat symbol to denote quantities associated to the physical spacetime in order to avoid its use later.

5 The advatadge of working with $\theta$ rather than with $A$ directly is that the former is manifestly gauge invariant, while $A$ is not. It is customary in the literature to set $A^{\prime}=0$, but this can be misleading during the calculation given that intermediate formulas would not have a manifestly gauge-invariant form. 
be evaluated following its definition (see [6, 27] for details) and reads:

$$
Q\left(A, A^{\prime}\right)=-\frac{1}{8 \pi^{2}} \theta \wedge F .
$$

It is straightforward now to check that $p_{1}\left(F^{\prime}\right)=p_{1}(0)=0$. Then, by integrating (9) and applying Stokes Theorem we get

$$
\int_{M} p_{1}(F)=\int_{\mathcal{J}} i^{*} Q\left(A, A^{\prime}\right)=-\frac{1}{16 \pi^{2}} \int_{\mathcal{J}} \theta_{a} F_{b c} \epsilon^{a b c} d u d \mathbb{S}^{2},
$$

where the boundary of the unphysical manifold is $\partial M=\mathcal{J} ; i^{*}$ denotes the pullback of the inclusion map $i: \mathcal{J} \hookrightarrow M$; and $d u d \mathbb{S}^{2}$ is the canonical integration measure on $\mathcal{J} \approx \mathbb{R} \times \mathbb{S}^{2}$. Note that the RHS is manifestly gauge-invariant, as it must be in view of the LHS.

This result can be further simplified if we work in a Newman-Penrose basis [34]. The electromagnetic field has 6 physical degrees of freedom per spacetime point that can be described with 3 complex scalars (these are analogous to the Weyl scalars in the gravitational case [35]). These scalars are the components of the tensor $F_{a b}$ in a null tetrad $\left\{\ell^{a}, n^{a}, m^{a}, \bar{m}^{a}\right\}$. Without loss of generality, we can take $n^{a}$ such that it equals $\eta^{a b} \nabla_{b} \Omega$ at $\Omega=0$, i.e. such that it is normal to the hypersurface $\mathcal{J}$. Then $\ell^{a}$ is chosen as a null vector that satisfies $\ell^{a} n_{a}=-1 ;$ and $m^{a}, \bar{m}^{a}$ are complex conjugate null vector fields, taken such that their real and imaginary parts are tangential to 2 -spheres (hence orthogonal to $n^{a}$ and $\ell^{a}$ ), and normalized as $m^{a} \bar{m}_{a}=1$. In this null tetrad, the metric takes the form $\eta_{a b}=-2 n_{(a} \ell_{b)}+2 m_{(a} \bar{m}_{b)}$ and the three electromagnetic scalars are defined by:

$$
\begin{aligned}
\Phi_{2} & =F_{a b} n^{a} \bar{m}^{b}, \\
\Phi_{1} & =\frac{1}{2}\left[F_{a b} n^{a} l^{b}+F_{a b} m^{a} \bar{m}^{b}\right], \\
\Phi_{0} & =F_{a b} m^{a} l^{b} .
\end{aligned}
$$

If we restrict to smooth solutions of Maxwell equations, the Peeling Theorem [36] guarantees that in a neighborhood of $\mathcal{J}$ we can expand $\Phi_{i}(u, \Omega, \theta, \phi)=\Phi_{i}^{0}(u, 0, \theta, \phi)+\Omega \Phi_{i}^{1}(u, 0, \theta, \phi)+\ldots$, where $(u, \theta, \phi)$ are Bondi-Sachs coordinates adapted to $\mathcal{J}$ [37, 38]. Going back to the physical spacetime, it is not difficult to see that this condition requires $\Phi_{2} \sim \frac{1}{r}$, so $\Phi_{2}^{0}$ represents the two radiative degrees of freedom of the electromagnetic field (corresponding to real and imaginary parts of this complex number). If we further assume the same asymptotic behaviour for the electromagnetic potential [39, 40], $A_{a} \sim O(1 / r)$, then the two radiative degrees of freedom are encoded in the component $A_{2}:=A_{a} \bar{m}^{a}$. Indeed, using $F_{a b}=2 \nabla_{[a} A_{b]}$ and the above definitions for the scalars, one can see that $A_{a} n^{a}=0$ and $\phi_{2}^{0}=\partial_{u} A_{2}^{0}$ at $\mathcal{J}$. Then,

We are now in position to evaluate (11). The tangent space of $\mathcal{J}$ is spanned by $\left\{n^{a}, m^{a}, \bar{m}^{a}\right\}$, so $\epsilon^{a b c}=i 3 ! n m^{[a} \bar{m}^{c]}$.

$$
\begin{aligned}
\int_{M} p_{1}(F) & =\frac{-6 i}{16 \pi^{2}} \int_{\mathcal{J}} \theta_{a} F_{b c}\left(n^{[a} m^{b} \bar{m}^{c]}\right) d u d \mathbb{S}^{2} \\
& =\frac{-2 i}{16 \pi^{2}} \int_{\mathcal{J}} \theta_{a} F_{b c}\left(n^{a} m^{[b} \bar{m}^{c]}+m^{a} \bar{m}^{[b} n^{c]}+\bar{m}^{a} n^{[b} m^{c]}\right) d u d \mathbb{S}^{2} \\
& =\frac{1}{4 \pi^{2}} \int_{\mathcal{J}} d u d \mathbb{S}^{2}\left(\theta_{a} n^{a} \operatorname{Im} \Phi_{1}^{0}-\operatorname{Im}\left(A_{2}^{0} \bar{\Phi}_{2}^{0}-\bar{\delta} \alpha \bar{\Phi}_{2}^{0}\right)\right)
\end{aligned}
$$

where we used ${A^{\prime}}_{2}^{0}=\overline{\bar{\partial}} \alpha$. Recalling that $A_{a} n^{a}=0$ at $\mathcal{J}$, we get

$$
\int_{M} p_{1}(F)=-\frac{1}{4 \pi^{2}} \int_{\mathcal{J}} d u d \mathbb{S}^{2} \operatorname{Im}\left(A_{2}^{0} \bar{\Phi}_{2}^{0}-\overline{\widehat{\partial}} \alpha \bar{\Phi}_{2}^{0}\right) .
$$

Notice that the value of $\alpha$ is determined by the choice of gauge of $A_{2}^{0}$. In other words, under a gauge transformation $A_{2}^{0}$ transforms as $A_{2} \rightarrow A_{2}^{0}+\overline{\mathrm{\gamma}} \beta$, while $\alpha$ transforms as $\alpha \rightarrow \alpha+\beta$, so the role of $\alpha$ is to maintain gauge invariance in the full expression. In a specific gauge, $\alpha$ can be set to zero.

\section{B. Physical interpretation: circularly polarized electromagnetic waves}

Since $A_{2}^{0}\left(\right.$ or $\left.\Phi_{2}^{0}\right)$ is a complex number that encodes the two radiative degrees of freedom of the electromagnetic field, we see that the chiral anomaly for fermions is intrinsically related to the emission of electromagnetic waves. Which 
properties should these waves have in order to produce a non-trivial result? To understand the physical meaning of this result, let us expand the electromagnetic field in Fourier modes as 6

$$
\Phi_{2}^{0}(u, \theta, \phi)=\int_{-\infty}^{\infty} \frac{d \omega}{2 \pi} \Phi(\omega, \theta, \phi) e^{-i \omega u}=\int_{0}^{\infty} \frac{d \omega}{2 \pi}\left[\Phi_{R}(\omega, \theta, \phi) e^{-i \omega u}+\bar{\Phi}_{L}(\omega, \theta, \phi) e^{i \omega u}\right],
$$

where in the second equality we explicitly splitted the modes in terms of positive and negative definite frequencies: $\Phi_{R}(\omega):=\Phi(\omega)$ for $\omega>0$ while $\bar{\Phi}_{L}(-\omega):=\Phi(\omega)$ for $\omega<0$. The electromagnetic potential satisfies $\dot{A}_{2}=\Phi_{2}^{0}$, so we can write

$$
A_{2}(u, \theta, \phi)=\int_{0}^{\infty} \frac{d \omega}{2 \pi}\left[\frac{\Phi_{R}(\omega, \theta, \phi)}{-i \omega} e^{-i \omega u}+\frac{\bar{\Phi}_{L}(\omega, \theta, \phi)}{i \omega} e^{i \omega u}\right]+\beta(\theta, \phi),
$$

where the function $\beta(\theta, \phi)$ emerges as a constant of integration. Imposing $\Phi_{2}=0$, one concludes that $\beta=\bar{\partial} \alpha$. Plugging these formulas in (16), we get

$$
\int d^{4} x \sqrt{-g}\left\langle\nabla_{a} j_{5}^{a}\right\rangle=\frac{e^{2}}{4 \pi^{2}} \int d \mathbb{S}^{2} \int_{0}^{\infty} \frac{d \omega}{2 \pi \omega}\left(\left|\Phi_{R}(\omega, \theta, \phi)\right|^{2}-\left|\Phi_{L}(\omega, \theta, \phi)\right|^{2}\right) .
$$

Note that this formula is reminiscent of the phenomenon of level-crossing, discussed in the introduction. What is the physical meaning of these modes, $\Phi_{R}$ and $\Phi_{L}$ ? The electromagnetic field $\Phi_{2}^{0}$ is self-dual, which means that it can be written as $\Phi_{2}^{0}=(E+i B)$, where $E, B$ are the electric and magnetic fields, representing the two possible, linearly independent polarization directions of the electromagnetic field. Because of this, we can also write

$$
\Phi_{2}^{0}(u, \theta, \phi)=\int_{-\infty}^{\infty} \frac{d \omega}{2 \pi}(E(\omega, \theta, \phi)+i B(\omega, \theta, \phi)) e^{-i \omega u},
$$

from which we identify

$$
\begin{aligned}
\Phi_{R}(\omega) & =(E(\omega)+i B(\omega)), & \omega>0, \\
\bar{\Phi}_{L}(-\omega) & =(E(\omega)+i B(\omega)), & \omega<0 .
\end{aligned}
$$

The second equation implies $\Phi_{L}(\omega)=(\bar{E}(-\omega)-i B(-\omega))$, for $\omega>0$. Because $E(u), B(u)$ are real functions, we must have then $\bar{E}(-\omega)=E(\omega)$ and $\bar{B}(-\omega)=B(\omega)$, leading to $\Phi_{L}(\omega)=(E(\omega)-i B(\omega))$. Taking into account all this, and expanding the fields in spin-weight spherical harmonics of modes $(\ell, m)$, we finally arrive at

$$
\left\langle\hat{Q}_{5}(\text { scri }+)\right\rangle-\left\langle\hat{Q}_{5}(\text { scri- })\right\rangle=\int_{0}^{\infty} \frac{d \omega e^{2}}{8 \pi^{3} \omega} \sum_{\ell m}\left[\left|E^{\ell m}(\omega)+i B^{\ell m}(\omega)\right|^{2}-\left|E^{\ell m}(\omega)-i B^{\ell m}(\omega)\right|^{2}\right] \text {. }
$$

The RHS represents the difference in intensity between right- and left-handed circularly polarized electromagnetic waves reaching future null infinity, i.e. the Stokes V parameter. The LHS represents the amount of net helicity spontaneously created on the fermion field. We conclude that the emission of circularly polarized electromagnetic radiation implies the spontaneous creation of massless charged fermions (highly energetic electrons, for instance) with net helicity. The more right- or left-handed electromagnetic radiation the spacetime contains, the more left or right-handed massless fermions will be excited from the quantum vacuum.

\section{A concrete example: electric-magnetic oscillating dipole}

Consider an electric dipole of moment $p_{0}$ pointing in the z-direction, oscillating with frequency $\omega$. The electromagnetic vector potential is [41]

$$
\vec{A}_{a}^{E}=-\frac{p_{0} \omega}{4 \pi e^{2} r} \cos (\omega u) \nabla_{a} z
$$

On top of this, consider a magnetic dipole of moment $m_{0}$ located in the $x-y$ plane, oscillating with the same frequency $\omega$ but in opposite phase. In the radiation-zone approximation, the electromagnetic vector potential yields [41]

$$
\vec{A}_{a}^{M}=-\frac{m_{0} \omega}{4 \pi e^{2}} \sin (\omega u) \sin ^{2} \theta \nabla_{a} \phi
$$

\footnotetext{
6 The condition $\Phi_{2}^{0}(u \rightarrow \pm \infty) \rightarrow 0$ is required from the finiteness of energy flux across $\mathcal{J}, \int_{-\infty}^{+\infty} d u|\Phi(u, \theta, \phi)|^{2}<\infty$. This condition in turn requires that $\Phi_{2}^{0}$ belongs to $L^{2}(\mathbb{C})$, and its Fourier transform exists. Notice that $A_{2}^{0}$ does not necessarily decay at $u \rightarrow \pm \infty$, so its Fourier transform is not defined.
} 
One expects this configuration to provide a non trivial Chern-Pontryagin because the magnetic fields of both the electric and magnetic oscillating dipoles considered here are entangled, leading to a non-zero magnetic helicity [42].

To calculate (16), let us work in spherical coordinates $(u, r, \theta, \phi)$. A (conformal) Newman-Penrose basis can be constructed such that $m^{a}=\frac{q^{a b}}{\sqrt{2}}\left(\nabla_{b} \theta+i \sin \theta \nabla_{a} \phi\right)$, where $q_{a b}=\operatorname{diag}\left\{1, \sin ^{2} \theta\right\}$ is the standard metric on the unit, homogeneous 2-sphere. The radiative component of the total electromagnetic potential is:

$$
A_{2}^{0}=\bar{m}^{a} \vec{A}_{a}=\frac{\omega \sin \theta}{4 \pi e^{2} \sqrt{2}}\left(i m_{0} \sin (\omega u)+p_{0} \cos (\omega u)\right) .
$$

Then,

$$
\int_{\mathcal{J}^{+}} d u d \mathbb{S}^{2} \operatorname{Im}\left(A_{2}^{0} \partial_{u} \bar{A}_{2}^{0}\right)=-\frac{\omega^{3} m_{0} p_{0}}{16 \pi e^{4}}\left(u_{2}-u_{1}\right)
$$

where $u_{2}-u_{1}$ is the period of (finite) time during which the system operates [we let $\omega \rightarrow 0$ for $u<u_{1}$ and $u>u_{2}$ ]. This result is gauge-invariant, so $\alpha=0$ in (16).

\section{THE GRAVITATIONAL CASE}

The fact that net fermion helicity can be spontaneously created from the quantum vacuum in a background of circularly polarized electromagnetic waves is a physically interesting result. In particular, it suggests that a similar phenomenon may occur for photon helicity in a background of gravitational waves, by simply noticing the parallelism with the chiral electromagnetic anomaly of (3). In this section we prove this in detail, following a similar strategy as in the electromagnetic case.

\section{A. Setup and main calculation}

Let $\left(\hat{M}, \hat{g}_{a b}\right)$ denote our physical, curved spacetime. The quantity of interest is

$$
\left\langle\hat{Q}_{5}(s c r i+)\right\rangle-\left\langle\hat{Q}_{5}(s c r i-)\right\rangle=\frac{\hbar}{48 \pi^{2}} \int_{\hat{M}} \operatorname{Tr}(\hat{R} \wedge \hat{R})=\frac{-\hbar}{96 \pi^{2}} \int_{\hat{M}} d^{4} x \sqrt{-g} \hat{R}_{a b c d}{ }^{a b c d}
$$

where $\hat{R}=\frac{1}{2} \hat{R}_{a b} d x^{a} \wedge d x^{b}$ is the curvature 2-form. Our goal is to compute this integral in an astrophysically relevant setting in order to know under which circumstances a given gravitational system may generate a flux of photons with net helicity. In particular, we restrict to asymptotically flat spacetimes. As in the previous section, the above integral is identically zero for stationary spacetimes. The proof is similar to the electromagnetic case but technically more tedious, so it is relegated to Appendix A. We must then focus on dynamical solutions of Einstein's equations and, to guarantee convergence, we consider spacetimes that asymptotically reach stationary regimes both at future and past timelike infinities. An example of this is a binary merger of two black holes which, ideally, are initially separated an infinite distance away, and end up merging to form a final stationary Kerr black hole.

As in the electromagnetic case, it is convenient to work instead with a conformally compactified spacetime, ( $M$, $\left.g_{a b}\right)$, constructed from the physical one by the standard procedure: $M=\hat{M} \cup \mathcal{J}, g_{a b}=\Omega^{2} \hat{g}_{a b}$. Our physical spacetime will be globally hyperbolic, so that $\hat{M} \simeq \mathbb{R} \times \Sigma\left[30\right.$, and in particular we shall restrict to $\Sigma \simeq \mathbb{R}^{3}$ (physically one does not expect more sophisticated spaces). The next step is to carry the relevant tensors of the physical spacetime to the unphysical one. It is useful to note that, due to the totally antisymmetric tensor $\hat{\epsilon}^{a b m n}$, the Ricci part in the physical Riemann tensor, $\hat{R}_{a b c}{ }^{d}$, does not contribute in this problem:

$$
\sqrt{\hat{g}} \hat{R}_{a b c}{ }^{d} \hat{\epsilon}^{a b m n} \hat{R}_{m n d}^{c}=\sqrt{\hat{g}} \hat{C}_{a b c}{ }^{d} \hat{\epsilon}^{a b m n} \hat{C}_{m n d}{ }^{c},
$$

where $\hat{C}_{a b c d}$ is the physical Weyl tensor. Using now the conformal invariance of the Weyl tensor, $\hat{C}_{a b c}{ }^{d}=C_{a b c}{ }^{d}$, that $\sqrt{-\hat{g}}=\Omega^{-4} \sqrt{-g}$, and that $\hat{\epsilon}^{a b c d}=\Omega^{4} \epsilon^{a b c d}$ then the quantity of interest turns out to be conformal invariant:

$$
\sqrt{\hat{g}} \hat{R}_{a b c}{ }^{d} \hat{\epsilon}^{a b m n} \hat{R}_{m n d}^{c}=\sqrt{g} R_{a b c}{ }^{d} \epsilon^{a b m n} R_{m n d}{ }^{c}
$$

and therefore

$$
\left\langle\hat{Q}_{5}(s c r i+)\right\rangle-\left\langle\hat{Q}_{5}(s c r i-)\right\rangle=\frac{\hbar}{48 \pi^{2}} \int_{\hat{M}} \operatorname{Tr}(\hat{R} \wedge \hat{R})=\frac{\hbar}{48 \pi^{2}} \int_{M} \operatorname{Tr}(R \wedge R) .
$$


Mathematically, $p_{1}(F)=-\frac{1}{8 \pi^{2}} \operatorname{Tr}(R \wedge R)$ is another invariant polynomial. To calculate this quantity we shall recall again the Chern-Weil Theorem. This theorem tells us that the difference between two invariant polynomials, $p_{1}(R)-p_{1}\left(R^{\prime}\right)$, associated to any two given connection 1-forms, $\omega$ and $\omega^{\prime}$ in $M$, is exact and is determined by the transgression term $Q\left(\omega, \omega^{\prime}\right)[\underline{6}]$ :

$$
p_{1}(R)-p_{1}\left(R^{\prime}\right)=d Q\left(\omega, \omega^{\prime}\right) .
$$

If we introduce the difference $\theta=\omega-\omega^{\prime}$, then the RHS can be evaluated in the standard way and yields [27]:

$$
Q\left(\omega, \omega^{\prime}\right)=-\frac{1}{8 \pi^{2}} \operatorname{Tr}\left(2 \theta \wedge R+\frac{2}{3} \theta \wedge \theta \wedge \theta-2 \theta \wedge \omega \wedge \theta-\theta \wedge d \theta\right) .
$$

Because the physical spacetime manifold is $\hat{M} \simeq \mathbb{R}^{4}$, it admits a flat Minkowskian metric $\hat{\eta}_{a b}$. Its associated conformal compactification, $\left(\mathbb{R}^{4} \cup \mathcal{J}^{+}, \eta_{a b}\right)$, with $\eta_{a b}=\Omega^{2} \hat{\eta}_{a b}$, will represent our auxiliary spacetime in this calculation. We denote all associated quantities with prime indices. Given that $\hat{C}^{\prime}{ }_{a b c}{ }^{d}=0$ in $\left(\mathbb{R}^{4}, \hat{\eta}_{a b}\right)$ one has $C^{\prime}{ }_{a b c}{ }^{d}=0$ at any point of $\mathbb{R}^{4} \cup \mathcal{J}^{+}$due to conformal invariance and continuity, and from (27) we deduce $p_{1}\left(R^{\prime}\right)=p_{1}\left(C^{\prime}\right)=p_{1}(0)=0$. Thus, the value of $\int_{M} p_{1}(R)$ is simply determined by the flux $Q\left(\omega, \omega^{\prime}\right)$ at future null infinity (i.e. at $\left.\Omega=0\right)$ :

$$
\int_{M} p_{1}(R)=\int_{\mathcal{J}^{+}} i^{*} Q\left(\omega, \omega^{\prime}\right)=-\frac{1}{8 \pi^{2}} \int_{\mathcal{J}^{+}} i^{*} \operatorname{Tr}\left(2 \theta \wedge R+\frac{2}{3} \theta \wedge \theta \wedge \theta-2 \theta \wedge \omega \wedge \theta-\theta \wedge d \theta\right) .
$$

The previous formula can be simplified in a more convenient manner. First note that $\theta \wedge d \theta=\theta \wedge d \omega-\theta \wedge d \omega^{\prime}=$ $\theta \wedge R-\theta \wedge \omega \wedge \omega+\theta \wedge \omega^{\prime} \wedge \omega^{\prime}$ (recall $R^{\prime}=d \omega^{\prime}+\omega^{\prime} \wedge \omega^{\prime}=0$ for Minkowski). Then

$$
\begin{aligned}
\operatorname{Tr}(-2 \theta \wedge \omega \wedge \theta-\theta \wedge d \theta) & =\operatorname{Tr} \theta \wedge\left(-2 \omega \wedge \theta+\omega \wedge \omega-\omega^{\prime} \wedge \omega^{\prime}\right)-\operatorname{Tr} \theta \wedge R \\
& =-\operatorname{Tr} \theta \wedge\left(\omega \wedge \omega-2 \omega \wedge \omega^{\prime}+\omega^{\prime} \wedge \omega^{\prime}\right)-\operatorname{Tr} \theta \wedge R \\
& =-\operatorname{Tr} \theta \wedge\left(\omega-\omega^{\prime}\right) \wedge\left(\omega-\omega^{\prime}\right)-\operatorname{Tr} \theta \wedge R,
\end{aligned}
$$

where in the last step we noticed that $\operatorname{Tr} \theta \wedge \omega \wedge \omega^{\prime}=\operatorname{Tr} \theta \wedge \omega^{\prime} \wedge \omega$. Eq. (32) can now be written as

$$
\int_{M} p_{1}(R)=\int_{\mathcal{J}^{+}} i^{*} Q\left(\omega, \omega^{\prime}\right)=-\frac{1}{8 \pi^{2}} \int_{\mathcal{J}^{+}} i^{*} \operatorname{Tr}\left(\theta \wedge R-\frac{1}{3} \theta \wedge \theta \wedge \theta\right) .
$$

It is convenient to introduce a $3+1$ splitting of $\left(M, g_{a b}\right)$ by $\{\Omega=$ const $\}$ hypersurfaces in order to simplify the integrand. Let $\hat{n}_{a}=\frac{1}{\sqrt{g^{a b} n_{a} n_{b}}} n_{a}$, with $n_{a}:=\nabla_{a} \Omega$, be the normalized transversal vector to the $\Omega=$ const hypersurfaces. The induced metric on these hypersurfaces is $h_{a b}=-\hat{n}_{a} \hat{n}_{b}+g_{a b}$, and its associated Levi-Civita derivative operator will be denoted by $D_{a}$. For any two vectors $u^{a}$, $v^{a}$ that are tangent to $\{\Omega=$ const $\}$ we can write the decomposition:

$$
u^{a} D_{a} v^{b}=u^{a} h_{c}^{b} \nabla_{a} v^{c}=u^{a}\left(g_{c}^{b}-\hat{n}^{b} \hat{n}_{c}\right) \nabla_{a} v^{c}=u^{a} \nabla_{a} v^{b}+u^{a}\left(\nabla_{a} \hat{n}_{c}\right) \hat{n}^{b} v^{c}
$$

where in the last equality $v^{a} \hat{n}_{a}=0$ was used. This leads to $D_{a} v_{b}=h_{a}^{c} \nabla_{c} v_{b}+\hat{n}_{b} v^{c} K_{a c}$, where $K_{a c}=D_{a} \hat{n}_{c}$ is the extrinsic curvature of $\{\Omega=$ const $\}$ as a hypersurface embedded in $M$ (as usual, it satisfies $K_{a c}=K_{c a}$ and $K_{a b} \hat{n}^{b}=0$, as can be easily checked). Consider now an orthonormal frame $\left\{e_{I}^{a}\right\}_{I=0, \ldots, 3}$ in $\left(M, g_{a b}\right)$, i.e. a set of 4 vectors labelled by $I$ that at each point $x$ of $M$ satisfy $g_{a b}(x) e_{I}^{a}(x) e_{J}^{b}(x)=\eta_{I J}$. The dual frame is defined via $e_{a, I}=g_{a b} e_{I}^{b}$, and latin indices $I, J, \ldots$ can be lowered and raised with $\eta_{I J}$. It is convenient to choose this frame as a Newman-Penrose tetrad $\left(\eta_{01}=\eta_{10}=-\eta_{23}=-\eta_{32}=-1\right.$, zero otherwise) such that for $\Omega=0$ the tangent space at future null infinity is spanned by $\left\{n^{a}, m^{a}, \bar{m}^{a}\right\}$. Given this tetrad, a (torsion-free) connection 1-form $\omega_{a}$ is defined by the equation $\nabla_{a} e_{b}^{I}+\omega_{a}^{I}{ }_{J} e_{b}^{J}=0$, and the metric-compatibility condition $\nabla_{a} g_{b c}=0$ gives the antisymmetry property $\omega_{a}^{I J}=-\omega_{a}^{J I}$. Taking $v_{b}=e_{b}^{I}$ in (35) we find

$$
h_{a}^{d}\left(\omega_{d}\right)_{I J}=-e_{J}^{b} D_{a} e_{b I}+K_{a I} \hat{n}_{b} e_{J}^{b}
$$

where $K_{a J}$ is a shorthand for $K_{a c} e_{J}^{c}$. Using the antisymmetry of $\omega_{a}^{I J}$ between $I$ and $J$, one can deduce: $h_{a}^{d}\left(\omega_{d}\right)^{I}{ }_{J}=$ $-\delta_{K}^{I}\left(e_{J}^{b} D_{a} e_{b}^{K}\right)-K_{a J} \hat{n}^{I}+K_{a}^{I} \hat{n}_{J}$, where $\delta_{J}^{I}$ and $\hat{n}^{I}$ are shorthands for $h_{a b} e^{a I} e_{J}^{b}$ and $\hat{n}^{a} e_{a}^{I}$. Let us introduce the additional notation $\left({ }^{3} \omega_{a}\right)^{I} \equiv-\delta_{K}^{I}\left(e_{J}^{b} D_{a} e_{b}^{K}\right)$. Thus

$$
\left(\omega_{a}\right)_{J}^{I}=\left({ }^{3} \omega_{a}\right)_{J}^{I}-K_{a J} \hat{n}^{I}+K_{a}^{I} \hat{n}_{J}+\hat{n}_{a} \hat{n}^{b}\left(\omega_{b}\right)_{J}^{I}
$$

Repeating this procedure exactly in the auxiliary Minkowskian spacetime $\left(\mathbb{R}^{4} \cup \mathcal{J}^{+}, \eta_{a b}\right)$ we get $\left(\omega_{a}^{\prime}\right)^{I}{ }_{J}=\left({ }^{3} \omega_{a}^{\prime}\right)^{I}{ }_{J}-$ $K_{a J}^{\prime} \hat{n}^{\prime I}+K^{\prime I}{ }_{a} \hat{n}_{J}^{\prime}+\hat{n}_{a}^{\prime} \hat{n}^{\prime b}\left(\omega_{b}^{\prime}\right)_{J}^{I}$, where prime indices denote quantities defined with respect to the metric $\eta_{a b}$. Taking the difference between the two (note that $h_{b}^{a} \hat{n}_{a}=h_{b}^{a} \hat{n}_{a}^{\prime}=0$ ),

$$
h_{a}^{d}\left(\theta_{d}\right)^{I J}=\left({ }^{3} \theta_{a}\right)^{I J}-2\left(K_{a}^{[J} \hat{n}^{I]}-K_{a}^{\prime[J}{\hat{n^{\prime}}}^{I]}\right) .
$$


As discussed in [31, 32] and summarized in Appendix B, $\mathcal{J}=\{\Omega=0\}$ is a three-dimensional null hypersurface that is endowed with a universal geometric structure, which consists in a collection of pairs $\left(\mathrm{g}_{a b}, \underline{\mathrm{n}}^{a}\right)$ satisfying a set of properties. Each pair consists of a degenerate metric $\mathrm{g}_{a b}$ on $\{\Omega=0\}$ and the corresponding null normal $\underline{\mathrm{n}}^{a}$. This geometric structure is common and available for any asymptotically flat spacetime. Consequently, we can fix the same conformal frame $\left(\mathrm{g}_{a b}, \underline{\mathrm{n}}^{a}\right)$ for both $\left(\mathbb{R}^{4} \cup \mathcal{J}^{+}, g_{a b}\right)$ and $\left(\mathbb{R}^{4} \cup \mathcal{J}^{+}, \eta_{a b}\right)$ spacetimes. Furthermore, without loss of generality we can choose this conformal frame such that $i^{*}\left(\Omega^{-2} g_{a b} n^{a} n^{b}\right)=1,\left(i^{*}\right.$ being the pullback of $\left.i: \mathcal{J} \hookrightarrow M\right)$ which will allow some simplifications in the next calculation. On the other hand, because the specification of the degenerate metric $\mathrm{g}_{a b}$ is equivalent to the specification of two complex-conjugate vectors $m^{a}, \bar{m}^{a}$ whose real and imaginary parts are tangential to the sphere, fixing this conformal frame $\left(\mathrm{g}_{a b}, \underline{\mathrm{n}}^{a}\right)$ is equivalent to fixing a common basis $\left\{n^{a}, m^{a}, \bar{m}^{a}\right\}$ for both spacetimes. Consequently, the two tetrads introduced above, $e_{I}^{a}$ and $e_{I}^{\prime a}$, agree for $\Omega=0$. Taking this into account and the orthogonality properties, $\left({ }^{3} \omega_{a}\right)^{I}{ }_{J} \hat{n}^{b} e_{b}^{J}=\left({ }^{3} \omega_{a}^{\prime}\right)^{I}{ }_{J}{\hat{n^{\prime}}}^{b} e_{b}^{\prime J}=K_{a J} \hat{n}^{b} e_{b}^{J}=K_{a J}^{\prime} \hat{n}^{\prime b} e_{b}^{\prime J}=0$, one finds

$$
i^{*} \operatorname{Tr} \theta \wedge \theta \wedge \theta=i^{*}\left[\left({ }^{3} \theta_{a}\right)^{I}{ }_{J}\left({ }^{3} \theta_{b}\right)^{J}{ }_{K}\left({ }^{3} \theta_{c}\right)_{I}^{K}-3\left({ }^{3} \theta_{a}\right)^{I J}\left(K_{b I}-K_{b I}^{\prime}\right)\left(K_{c J}-K_{c J}^{\prime}\right)\right] \epsilon^{a b c} \sqrt{h} d^{3} x
$$

and

$$
\left.i^{*} \operatorname{Tr} \theta \wedge R=\frac{1}{2} i^{*}\left[\left({ }^{3} \theta_{a}\right)_{I}^{J} R_{b c}{ }^{I}{ }^{\prime}-2\left(K_{a I} \hat{n}^{J}-K^{\prime}{ }_{a I}{\hat{n^{\prime}}}^{J}\right) R_{b c}{ }^{I}\right]\right] \epsilon^{a b c} \sqrt{h} d^{3} x .
$$

The 1-form $\left({ }^{3} \theta_{a}\right)^{I}{ }_{J}$ can be determined at $\Omega=0$ from the intrinsic geometry of future null infinity. As discussed in Appendix B, for any covector $e_{b}^{I}$ at null infinity, the difference $D_{a}^{\prime}-D_{a}$ between two (equivalence classes of) connections is completely characterized by a traceless, symmetric tensor $\sigma_{a b}$ : $\left(D_{a}-D_{a}^{\prime}\right) e_{b}^{I}=-\sigma_{a b} n^{c} e_{c}^{I}$. Since, as discussed above, at $\Omega=0$ the tetrad $e_{a}^{I}$ is equal to the tetrad $e^{\prime I}$, and $D_{a}$ is tangential to $\Omega=0$, we have $\left({ }^{3} \theta_{a}\right)^{I}{ }_{J}=-\delta_{K}^{I} e_{J}^{b}\left(D_{a}-D_{a}^{\prime}\right) e_{b}^{K}$ at $\Omega=0$, and hence $\left({ }^{3} \theta_{a}\right)^{I}{ }_{J} e_{I}^{d} e_{e}^{J}=-e_{I}^{d} \delta_{K}^{I}\left(D_{a}-D_{a}^{\prime}\right) e_{e}^{K}=-e_{I}^{d} \delta_{K}^{I} \sigma_{a e} n^{g} e_{g}^{K}$ at $\Omega=0$. Now: $e_{I}^{d} \delta_{K}^{I} n^{g} e_{g}^{K}=e_{I}^{d}\left(\eta_{K}^{I}-\hat{n}^{I} \hat{n}_{K}\right) n^{g} e_{g}^{K}=n^{d}-\hat{n}_{g} n^{g} \hat{n}^{d}=n^{d}-\frac{n^{g} \nabla_{g} \Omega g^{d b} \nabla_{b} \Omega}{g^{e f} \nabla_{e} \Omega \nabla_{f} \Omega}$, and since at $\Omega=0$ we have $n^{a}=g^{a b} \nabla_{b} \Omega$, we conclude

$$
i^{*}\left[\left({ }^{3} \theta_{a}\right)^{I}{ }_{J} e_{I}^{d} e_{e}^{J}\right]=0
$$

and we are led to

$$
\int_{M} p_{1}(R)=\frac{1}{8 \pi^{2}} \int_{\mathcal{J}^{+}} i^{*}\left[\left(K_{a d}-K_{a d}^{\prime}\right) \hat{n}^{e} R_{b c}{ }^{d} \epsilon^{a b c} \sqrt{h} d^{3} x\right] .
$$

We obtain now a compact expression for the extrinsic curvature of $\mathcal{J}^{+}$as a hypersurface of $M$. First note that

$$
i^{*}\left(K_{a b}-K_{a b}^{\prime}\right)=i^{*}\left(D_{a} \hat{n}_{b}-D_{a}^{\prime} \hat{n}_{b}^{\prime}\right)=i^{*}\left[\left(D_{a} \alpha\right) n_{b}+\alpha D_{a} n_{b}-\left(D_{a}^{\prime} \alpha^{\prime}\right) n_{b}-\alpha^{\prime} D_{a}^{\prime} n_{b}\right]=i^{*}\left(\Omega^{-1}\left(D_{a} n_{b}-D_{a}^{\prime} n_{b}\right)\right),
$$

where we denoted $\alpha \equiv \frac{1}{\sqrt{g^{a b} \nabla_{a} \Omega \nabla_{b} \Omega}}$ for brevity and in the last step we took into account that $i^{*}(\Omega \alpha)=i^{*}\left(\Omega \alpha^{\prime}\right)=1$. The term inside parenthesis vanishes as $O(\Omega)$ at null infinity but the prefactor diverges as $\Omega^{-1}$, so the product is a well defined, smooth quantity at null infinity. To calculate its value let us use equation (B4) from Appendix B:

$$
\Omega S_{a b}+2 \nabla_{a} n_{b}-\Omega^{-1} n^{c} n_{c} g_{a b}=O\left(\Omega^{3}\right) .
$$

The pull-back of $i: \mathcal{J}^{+} \rightarrow M$ on this expression provides us with the value of $\Omega^{-1} \nabla_{a} n_{b}$ at future null infinity,

$$
i^{*}\left(\Omega^{-1} \nabla_{a} n_{b}\right)=-\frac{1}{2} \underline{\mathrm{S}}_{a b}+\frac{1}{2} \underline{\underline{g}}_{a b} i^{*}\left(\Omega^{-2} n^{a} n_{a}\right)=-\frac{1}{2} \underline{\mathrm{S}}_{a b}+\frac{1}{2} \mathrm{~g}_{a b} .
$$

Repeating the same with the auxiliary Minkowski space, one gets (recall that we fixed the same conformal frame $\left(\mathrm{g}_{a b}, \underline{\mathrm{n}}^{c}\right)$ for both spacetimes):

$$
i^{*}\left(\Omega^{-1} \nabla_{a} n_{b}\right)-i^{*}\left(\Omega^{-1} \nabla_{a}^{\prime} n_{b}\right)=i^{*}\left(\Omega^{-1}\left(D_{a} n_{b}-D_{a}^{\prime} n_{b}\right)\right)=-\frac{1}{2}\left(S_{a b}-S_{a b}^{\prime}\right) .
$$

But for Minkowski, $S_{a b}^{\prime}=\rho_{a b}$, where $\rho_{a b}$ is the "gauge" part of $S_{a b}$. The combination $S_{a b}-\rho_{a b}=: N_{a b}$ is manifestly invariant under conformal gauge transformations of the form $\Omega \rightarrow \omega \Omega$, and defines what is known as the Bondi News tensor, $N_{a b}$ (see Appendix B). This is the quantity that determines whether an asymptotically flat spacetime contains non-trivial gravitational radiation. We find

$$
i^{*}\left(K_{a b}-K_{a b}^{\prime}\right)=-\frac{1}{2} N_{a b} .
$$


On the other hand:

$$
i^{*}\left(\hat{n}^{e} R_{b c}{ }^{d} e^{\epsilon^{a b c} \sqrt{h}}\right)=i^{*}\left(n^{e} \Omega^{-1}\left(C_{b c e}{ }^{d}+g_{e[b} S_{c]}^{d}-\delta_{[b}^{d} S_{c] e}\right) \epsilon^{a b c} \sqrt{h}\right) .
$$

Because $\hat{n}_{a} \epsilon^{a b c}=0$, the second term does not contribute. On the other hand, the third term can be written as $i^{*}\left(\hat{n}^{e} S_{e}^{n} g_{c n} \epsilon^{a d c}\right)=i^{*}\left(g_{c n} \epsilon^{a d c} \Omega^{-1}\right) i^{*}\left(n^{e} S_{e}^{n}\right) \propto i^{*}\left(g_{c b} \epsilon^{a d c} \Omega^{-1}\right) i^{*}\left(n^{b}\right)=i^{*}\left(\epsilon^{a d c}\right) i^{*}\left(\Omega^{-1} n^{b} g_{b c}\right)=0$, where we used $S_{b}^{a} n^{b} \propto n^{a}$ and $\underline{\mathrm{n}}^{a} i^{*}\left(\Omega^{-2} n_{a}\right)=1$ in the last step. Thus,

$$
\begin{aligned}
i^{*}\left(\hat{n}^{e} R_{b c}{ }^{d} e^{\left.\epsilon^{a b c} \sqrt{h}\right)}\right. & =i^{*}\left(n^{e} \Omega^{-1} C_{b c e}{ }^{d} \epsilon^{a b c} \sqrt{h}\right) \\
& =-i^{*}\left(n_{e} \Omega^{-1} C_{b c}^{* *} e d \epsilon^{a b c} \sqrt{h}\right) \\
& =\frac{1}{2} i^{*}\left(\epsilon^{d p q e} n_{e} \Omega^{-1} C_{b c p q}^{*} \epsilon^{a b c} \sqrt{h}\right),
\end{aligned}
$$

where $*$ denotes the Hodge dual. Note that $\epsilon^{a b c}=\epsilon^{a b c d} \hat{n}_{d}=\Omega^{-1} \epsilon^{a b c d} n_{d}$ but $\sqrt{h}=\Omega \sqrt{q}$, where $q_{a b}$ is the metric of the two-dimensional spheres, so that $\epsilon^{a b c} \sqrt{h}=\epsilon^{a b c d} n_{d} \sqrt{q}$. Now, the quantity $\left(\epsilon^{d p q e} n_{e}\right)\left(\epsilon^{a b c m} n_{m}\right)\left(\Omega^{-1} C_{b c p q}^{*}\right)$ is smooth in all $M$, and thus it exists in $\mathcal{J}^{+}$(see Appendix B). Its pullback to null infinity is denoted as ${ }^{*} K^{a d}$. Taking into account all this and using ${ }^{*} K^{a b}=2 \epsilon^{p q a} D_{p} N_{q}{ }^{b}[31]$ :

$$
i^{*}\left(K_{a d}-K^{\prime}{ }_{a d}\right) i^{*}\left(\hat{n}^{e} R_{b c}{ }^{d} \epsilon^{a b c} \sqrt{h}\right)=-\frac{1}{4} N_{m n}{ }^{*} K^{m n} \sqrt{q}=-\frac{1}{2} N_{m n} \epsilon^{p q m} D_{p} N_{q}{ }^{n} \sqrt{q} .
$$

This expression can be simplified further. The basis $\left\{n^{a}, m^{a}, \bar{m}^{a}\right\}$ satisfies $0=\mathcal{L}_{n} m^{a}=n^{d} D_{d} m^{a}$. Using $\epsilon^{a b c}=$ $i 3 ! n^{[a} m^{b} \bar{m}^{c]}$, and $N_{a b} n^{b}=0, D_{a} n^{b}=0$, we can get

$$
i^{*}\left(K_{a d}-K_{a d}^{\prime}\right) i^{*}\left(\hat{n}^{e} R_{b c}{ }^{d} e^{a b c} \sqrt{h}\right)=-i N_{a b} m^{[n} \bar{m}^{a]} n^{d} D_{d} N_{n}^{b}=-\operatorname{Im}\left(N_{44} \partial_{u} N_{33}\right),
$$

where $N_{33}:=N_{a b} m^{a} m^{b}$ and $N_{44}=\bar{N}_{33}$, following the usual Newman-Penrose notation.

Taking into account these results, we can rewrite (29) in the final form

$$
\int d^{4} x \sqrt{-g}\left\langle\nabla_{a} j_{5}^{a}\right\rangle=-\frac{\hbar}{6} \int_{M} p_{1}(R)=\frac{\hbar}{48 \pi^{2}} \int_{\mathcal{J}^{+}} d u d \mathbb{S}^{2} \operatorname{Im}\left(N_{44} \partial_{u} N_{33}\right) .
$$

Note the strong analogy with the electromagnetic case, equation (16), and also that this result is manifestly gauge invariant ( $N_{a b}$ is invariant under conformal gauge transformations of the form $\Omega \rightarrow \omega \Omega$ ). On the other hand, notice that this result is purely geometrical. In other words, the topological information encoded in the Chern-Pontryagin is here trivial (zero) because we are just working with $\mathbb{R}^{4}$ with the usual differentiable structure. It is the contribution of the boundary (physically, null infinity) what contributes non-trivially to the final result, but this contribution is not topological. For manifolds with boundary, the Chern-Pontryagin is not purely topological, and its utility as a topological invariant is recovered only when a surface correction is added [27]. This correction is precisely equal to the result that we obtain with a sign reversed.

\section{B. Physical interpretation: circularly polarized gravitational waves}

The result (51) tells us that the electromagnetic duality anomaly (4) is fully determined by the radiative content of the spacetime. To write the result in terms of the $\Psi_{4}^{0}$ Weyl scalar, widely used in the gravitational-wave literature, we notice that $N_{33}=2 \dot{\sigma}$ and $\Psi_{4}^{0}=-\ddot{\bar{\sigma}}$, where $\sigma$ is the shear of the gravitational radiation (see Appendix C). Then,

$$
\int d^{4} x \sqrt{-g}\left\langle\nabla_{a} j_{5}^{a}\right\rangle=\frac{\hbar}{12 \pi^{2}} \int_{\mathcal{J}^{+}} d u d \mathbb{S}^{2} \int^{u} d u^{\prime} \operatorname{Im}\left(\Psi_{4}^{0}\left(u^{\prime}, \theta, \phi\right) \bar{\Psi}_{4}^{0}(u, \theta, \phi)\right) .
$$

Despite its apparent form, the physical interpretation of this result is remarkably simple. Expand in Fourier modes as

$$
\Psi_{4}^{0}(u, \theta, \phi)=\int_{-\infty}^{\infty} \frac{d \omega}{2 \pi} h(\omega, \theta, \phi) e^{-i \omega u}=\int_{0}^{\infty} \frac{d \omega}{2 \pi}\left[h_{R}(\omega, \theta, \phi) e^{-i \omega u}+\bar{h}_{L}(\omega, \theta, \phi) e^{i \omega u}\right],
$$

where in the second equality we splitted the modes explicitly in terms of positive and negative definite modes: $h_{R}(\omega):=h(\omega)$ for $\omega>0$ while $\bar{h}_{L}(-\omega):=h(\omega)$ for $\omega<0$. The News scalar satisfies $\dot{N}_{33}=-2 \bar{\Psi}_{4}^{0}$, so we can write

$$
N_{33}(u, \theta, \phi)=2 \int_{0}^{\infty} \frac{d \omega}{2 \pi}\left[\frac{h_{L}(\omega, \theta, \phi)}{i \omega} e^{-i \omega u}+\frac{\bar{h}_{R}(\omega, \theta, \phi)}{-i \omega} e^{i \omega u}\right]+\beta(\theta, \phi),
$$


where the function $\beta(\theta, \phi)$ emerges as a constant of integration. This function, however, does not contribute to (51) because the physical requirement of finite GW energy crossing null infinity implies $N_{33}(u \rightarrow \pm \infty) \rightarrow 0$ (this is inferred from the Bondi mass formula, [31]), and leads to $\beta(\theta, \phi)=0$. From (51),

$$
\int d^{4} x \sqrt{-g}\left\langle\nabla_{a} j_{5}^{a}\right\rangle=-\frac{\hbar}{12 \pi^{2}} \int d \mathbb{S}^{2} \int_{0}^{\infty} \frac{d \omega}{2 \pi \omega}\left(\left|h_{R}(\omega, \theta, \phi)\right|^{2}-\left|h_{L}(\omega, \theta, \phi)\right|^{2}\right) .
$$

What is the physical meaning of these modes, $h_{R}$ and $h_{L}$ ? Because $\Psi_{4}^{0}=-\ddot{\bar{\sigma}}=-\left(\ddot{h}_{+}-i \ddot{h}_{\times}\right)$, where $h_{+}, h_{\times}$are the two standard linear polarization modes of the GWs, we also have:

$$
\Psi_{4}^{0}(u, \theta, \phi)=\int_{-\infty}^{\infty} \frac{d \omega}{2 \pi} \omega^{2}\left(h_{+}(\omega, \theta, \phi)-i h_{\times}(\omega, \theta, \phi)\right) e^{-i \omega u}
$$

from which we identify

$$
\begin{array}{rlrl}
h_{R}(\omega) & =\omega^{2}\left(h_{+}(\omega)-i h_{\times}(\omega)\right), & \omega>0, \\
\bar{h}_{L}(-\omega) & =\omega^{2}\left(h_{+}(\omega)-i h_{\times}(\omega)\right), \quad \omega<0 .
\end{array}
$$

The second equation implies $h_{L}(\omega)=\omega^{2}\left(\bar{h}_{+}(-\omega)+i \bar{h}_{\times}(-\omega)\right)$, for $\omega>0$. Because $h_{+}(u), h_{\times}(u)$ are real functions, we must have $\bar{h}_{+}(-\omega)=h_{+}(\omega)$ and $\bar{h}_{\times}(-\omega)=h_{\times}(\omega)$, leading to $h_{L}(\omega)=\omega^{2}\left(h_{+}(\omega)+i h_{\times}(\omega)\right)$, and

$$
\left\langle Q_{5}(\text { scri }+)\right\rangle-\left\langle Q_{5}(\text { scri- })\right\rangle=\hbar \int_{0}^{\infty} \frac{d \omega \omega^{3}}{24 \pi^{3}} \sum_{\ell m}\left[\left|h_{+}^{\ell m}(\omega)+i h_{\times}^{\ell m}(\omega)\right|^{2}-\left|h_{+}^{\ell m}(\omega)-i h_{\times}^{\ell m}(\omega)\right|^{2}\right],
$$

where we expanded the field variables in spin-weight spherical harmonics of modes $(\ell, m)$. The physical interpretation of this result is again clear: while the LHS represents the net amount of photon circular polarization created, the RHS is the difference in intensity between right- and left-handed circularly polarized GWs reaching future null infinity, i.e. the Stokes V parameter of GWs. Thus, we conclude that the emission of chiral gravitational radiation by astrophysical systems implies the spontaneous creation of photons with net helicity. The more right- or left-handed GWs the spacetime contains, the more left or right-handed photons will be excited from the quantum vacuum.

\section{An example: precessing binary black hole systems}

Let us consider a binary black hole merger. The system emits GWs, which are analyzed in modes of frequency $\omega$ and angular numbers $(\ell, m)$. During the inspiral phase the frequency spectrum is determined by the angular velocity $\Omega$ as $\omega_{m} \sim m \Omega$. The shear of the gravitational waves can be decomposed as $\sigma(u, \theta, \phi)=$ $\sum_{\ell m}\left(A_{\ell m-2}^{+} Y_{\ell m}(\theta, \phi) e^{-i \omega_{m} u}+\bar{A}_{\ell m}^{-} \bar{Y}_{\ell m}(\theta, \phi) e^{i \omega_{m} u}\right)$. Self-consistency requires $\bar{A}_{\ell m}^{-}=(-1)^{m} A_{\ell-m}^{+}$, which is deduced using ${ }_{s} \bar{Y}_{l m}=(-1)^{s+m}{ }_{-s} Y_{l(-m)}$. Equation (58) gives

$$
\left\langle Q_{5}(s c r i+)\right\rangle-\left\langle Q_{5}(s c r i-)\right\rangle \propto \sum_{\ell m} \omega_{m}^{3}\left(\left|A_{\ell m}^{+}\right|^{2}-\left|A_{\ell(-m)}^{+}\right|^{2}\right) .
$$

The parameters $A_{\ell m}^{ \pm}$can be understood as "excitation" factors for the generation of each GW polarization of mode $(\ell, m)$, and they depend on the details of the physical system under consideration (initial data). If the binary system is invariant under mirror symmetry with respect to some plane, choosing angular coordinates such that $\theta=\pi / 2$ represents that plane, this invariance is equivalent to say that $C_{a b c d}(u, \theta, \phi)=C_{a b c d}(u, \pi-\theta, \phi)$, which implies $\sigma(u, \theta, \phi)=\bar{\sigma}(u, \pi-\theta, \phi)$. Using ${ }_{s} Y_{l m}(\pi-\theta, \phi+\pi)=(-1)^{l}{ }_{-s} Y_{l m}(\theta, \phi)$, the previous condition leads to $A_{\ell m}^{+}=A_{\ell(-m)}^{+}(-1)^{\ell}$, which makes (59) vanish. In other words, the chiral anomaly emerges in binary mergers that do not have any mirror symmetry 7 Examples of this are precessing binary systems, in which the individual spins of the BHs are not aligned with the total angular momentum [43] and break any potential symmetry under mirror transformations (see [29] for more details and implications in astrophysics).

\footnotetext{
7 Note that we are neglecting the backreaction of GWs on the evolution of the inspiral. When this is taken into account, the radius of the orbit shrinks for any binary merger, and to some extent this breaks the symmetry under spatial reversals. However, this process can be considered adiabatic, and its contribution insignificant.
} 


\section{CONCLUSIONS}

Chiral anomalies are a long-standing prediction of quantum field theory that have provided rich physical consequences along the last decades in several branches of physics. Despite this, their use has been considerably restricted to non-trivial topological issues, with instantons playing a dominant role. While this has been fruitful in many aspects, as for instance in unraveling the vacuum structure in Yang-Mills theories and solving problems of major importance in particle physics, it is not the whole story, at least in gravity (and electrodynamics). In this paper we characterized which class of solutions to Einstein's (and Maxwell) equations are able to induce the chiral anomaly on fermion and electromagnetic fields. On the one hand, we found that stationary solutions cannot trigger this anomaly. On the other hand we found that, among all dynamical solutions, only those which involve radiation with net circular polarization are able to induce the quantum anomaly, and we provided specific examples of physical interest where this occurs. The physical interpretation of this quantum effect is associated to spontaneous creation of particles, but in sharp contrast to the familiar Hawking radiation of black holes, a net amount of helicity can be originated from the quantum vacuum. This new aspect of chiral anomalies could be useful in the search for phenomenology, but this is out of the scope of this paper and will be left for future studies.

Acknowledgments.- The author is grateful to I. Agullo and J. Navarro-Salas for useful comments and many discussions over the time that this work took place, and to A. Ashtekar for useful discussions on the convergence of the integrals. The author acknowledges support under NSF grant PHY-1806356 and the Eberly Chair funds of Penn State; and funds from the grant No. FIS2017-91161-EXP during an early stage of this work.

\section{Appendix A: Stationary spacetimes}

In this appendix we prove that in stationary, asymptotically flat spacetimes $\left(M, g_{a b}\right)$ with $M \simeq\left(t_{1}, t_{2}\right) \times \mathbb{R}^{3}$ one has

$$
\int_{M} d^{4} x \sqrt{-g} R_{a b c d}^{*} R^{a b c d}=0 .
$$

The argument follows in close analogy to the electromagnetic case (see section ПA).

Given a local orthonormal frame ("vierbein") $\left\{e_{I}^{a}\right\}$, we can define the curvature 2-form from the Riemann tensor as $R_{a b}{ }^{I}{ }_{J}=R_{a b c}{ }^{d} e_{d}^{I} e_{J}^{c}$. For notational simplifity we will frequently omit the internal indices $I, J$ of the curvature 2 -form and/or work directly with $R=\frac{1}{2} R_{a b} d x^{a} \wedge d x^{b}$. If the spacetime is stationary there exists a timelike killing vector $k^{a}$ that leaves the metric invariant along its integral curves, $\mathcal{L}_{k} g_{a b}=0$. We construct our tetrad basis $\left\{e_{I}^{a}\right\}$ such that $\mathcal{L}_{k} e_{I}^{a}=0$ as well. The stationarity condition leads to $\mathcal{L}_{k} R_{a b c d}=0$. Together with the previous equation it gives $\mathcal{L}_{k} R_{a b}=0$, or equivalently $d i_{k} R+i_{k} d R=0$. For a general matrix-valued, $p$-form $V$ we can introduce the covariant derivative $D V=d V+\omega \wedge V-(-1)^{p} V \wedge \omega[27]$, under which the familiar Bianchi identity $\nabla_{a}^{*} R^{a b c d}=0$ is equivalent to $D R=d R+\omega \wedge R-R \wedge \omega=0$. Using these equations we can write $i_{k} d R=i_{k}(-\omega \wedge R+R \wedge \omega)=$ $-i_{k} \omega \wedge R+\omega \wedge i_{k} R+i_{k} R \wedge \omega+R \wedge i_{k} \omega$ and $d\left(i_{k} R\right)=D\left(i_{k} R\right)-\omega \wedge i_{k} R-i_{k} R \wedge \omega$. Joining both results:

$$
D\left(i_{k} R\right)=i_{k} \omega R-R i_{k} \omega .
$$

For any matrix $\Lambda$ one has $D(D \Lambda)=-\Lambda R+R \Lambda$, so one can deduce from the above that $i_{k} R=-D\left(i_{k} \omega\right)$, or $k^{a} R_{a b}=-\nabla_{b} i_{k} \omega$.

On the other hand, let us use the normalized vector $\hat{k}^{a}=\frac{1}{\alpha} k^{a}$, with $\alpha=\sqrt{-k^{a} k_{a}}$, to make a $3+1$ decomposition of the metric, $g_{a b}=-\hat{k}_{a} \hat{k}_{b}+h_{a b}$. This decomposition allows the simplification:

$$
\int_{M} d^{4} x \sqrt{-g} R_{a b c d}{ }^{*} R^{a b c d}=\operatorname{Tr} \int_{M} d^{4} x \sqrt{-g} R_{a b}{ }^{*} R^{a b}=-4 \operatorname{Tr} \int_{M} d^{4} x \sqrt{-g} \hat{k}^{a} R_{a b}{ }^{*} R_{c}{ }^{b} \hat{k}^{c},
$$

where in the last equality we used ${ }^{*} R_{a b}=\frac{1}{2} \epsilon_{a b c d} R^{c d}$ to write $\operatorname{Tr} h^{a b} h^{c d} R_{a c}{ }^{*} R_{b d}=-2 \operatorname{Tr} \hat{k}^{a} R_{a b} \hat{k}_{c}{ }^{*} R^{c b}$. Doing some manipulations one gets

$$
\operatorname{Tr}\left(\left(D_{b} i_{k} \omega\right) \alpha^{-1} \hat{k}_{c}{ }^{*} R^{c b}\right)=\nabla_{b} \operatorname{Tr}\left(i_{k} \omega \alpha^{-1} \hat{k}_{c}{ }^{*} R^{c b}\right)-\nabla_{b}\left(\alpha^{-1} \hat{k}_{c}\right) \operatorname{Tr}\left({ }^{*} R^{c b} i_{k} \omega\right) .
$$

It turns out that the second term is identically zero. To see this expand as

$$
\nabla_{b}\left(\alpha^{-1} \hat{k}_{c}\right)=\alpha^{-1} \nabla_{b} \hat{k}_{c}-\frac{1}{\alpha^{2}} \hat{k}_{c} \nabla_{b} \alpha .
$$


Since $\nabla_{b} \alpha=-\frac{1}{\alpha} k^{d} \nabla_{b} k_{d}$ and $\nabla_{a} k_{b}=-\nabla_{b} k_{a}\left(k^{a}\right.$ is a Killing Vector field):

$$
\nabla_{b}\left(\alpha^{-1} \hat{k}_{c}\right)=\alpha^{-1}\left(\nabla_{b} \hat{k}_{c}-\frac{1}{\alpha} \hat{k}_{c} \hat{k}^{d} \nabla_{d} k_{b}\right)=\frac{1}{\alpha}\left(2 \nabla_{(b} \hat{k}_{c)}-D_{c} \hat{k}_{b}\right),
$$

where in the last equality we used $k^{a} \nabla_{a} \alpha=0$ (which can be deduced after expanding $k^{a} \mathcal{L}_{k} k_{a}=k^{a} g_{a b} \mathcal{L}_{k} k^{b}=0$ ), $\hat{k}_{a} \hat{k}_{b}=-g_{a b}+h_{a b}$, and introduced the spatial covariant derivative $D_{a} \hat{k}_{b}=h_{a}^{c} h_{b}^{d} \nabla_{c} \hat{k}_{d}=h_{a}^{c} \nabla_{c} \hat{k}_{b}$. The RHS is a symmetric tensor, so when contracting with ${ }^{*} R^{c b}$ in (A4) the result will be zero.

We end up with the integral of a total derivative, which can be solved using Stokes Theorem. Let us work in coordinates $\{t, r, \theta, \phi\}$, where $t$ is the time measured by static observers at spatial infinity: $k \rightarrow \frac{\partial}{\partial t}$ as $r \rightarrow \infty$. Then

$$
\int_{M} d^{4} x \sqrt{-g} R_{a b c d}{ }^{*} R^{a b c d}=\lim _{r \rightarrow \infty} \int_{t_{1}}^{t_{2}} d t \int d \mathbb{S}^{2} r^{2} \operatorname{Tr}\left(i_{k} \omega \hat{k}_{c}{ }^{*} R^{c d} \nabla_{d} r\right) .
$$

(notice that for asymptotically flat spacetimes: $\alpha \rightarrow 1$ as $r \rightarrow \infty$ ). Using $\mathcal{L}_{k} e_{I}^{a}=\left[k, e_{I}\right]^{a}=0$ one can further deduce that $\left(i_{k} \omega\right)_{I J}=e_{I}^{a} e_{J}^{b} \nabla_{a} k_{b}$ so

$$
\int_{M} d^{4} x \sqrt{-g} R_{a b c d}{ }^{*} R^{a b c d}=\lim _{r \rightarrow \infty} \int_{t_{1}}^{t_{2}} d t \int d \mathbb{S}^{2} r^{2} \nabla_{a} k_{b}^{*} R^{a b c d} k_{c} \nabla_{d} r .
$$

At spatial infinity we have $\nabla_{a} k_{b}=\nabla_{a} \nabla_{b} t=0$ so $\nabla_{a} k_{b} \sim O\left(r^{-1}\right)$. Assuming standard fall-off conditions at spatial infinity for the Weyl tensor [44], $C_{a b c d} \sim O\left(r^{-3}\right)$, we finally see that

$$
\int_{M} d^{4} x \sqrt{-g} R_{a b c d}^{*} R^{a b c d}=0 .
$$

\section{Appendix B: Asymptotic Minkowskian spacetimes}

We summarize here the basic points of [31 33] that are needed to follow the calculation in the main text.

A spacetime $\left(\hat{M}, \hat{g}_{a b}\right)$ is called asymptotically flat at null infinity if there exists a manifold $M$ with boundary $I$ endowed with a metric tensor $g_{a b}$, and a diffeomorphism from $\hat{M}$ onto $M-I$ (with which we identify $\hat{M}$ and $M-I$ ) that satisfies:

(a) there exists a smooth function $\Omega$ on $M$ with $g_{a b}=\Omega^{2} \hat{g}_{a b}$ on $\hat{M} ; \Omega=0$ on $I$; and $n_{a}:=\nabla_{a} \Omega$ is non-vanishing at $I$.

(b) $I$ is homeomorphic to $\mathbb{S}^{2} \times \mathbb{R}$.

(c) $\tilde{g}_{a b}$ satisfies Einstein's equations $\hat{R}_{a b}-\frac{1}{2} \hat{R} \hat{g}_{a b}=8 \pi G \hat{T}_{a b}$, and $\Omega^{-2} \hat{T}_{a b}$ has a smooth limit to $I$.

One refers to $\left(\hat{M}, \hat{g}_{a b}\right)$ as the physical spacetime, and to $\left(M, g_{a b}\right)$ as the unphysical one, or the conformal completion of $\left(\hat{M}, \hat{g}_{a b}\right)$. Using the known conformal transformation rules for the Ricci tensor and scalar curvature, it is easy to find that these conditions imply $n^{a} n_{a}=0$ on $I$. Thus, $I$ is a 3 -dimensional null hypersurface in $M$.

Notice that within this definition there is freedom to perform conformal rescalings: if $\Omega$ is an allowed conformal factor for a physical spacetime $\left(\hat{M}, \hat{g}_{a b}\right)$, so is $\Omega^{\prime}=\omega \Omega$, where $\omega$ is a smooth function on $M$ and non-vanishing at $I$. Under this conformal gauge transformation, it is easy to check that $g_{a b} \rightarrow \omega^{2} g_{a b}, n^{a} \rightarrow \omega^{-1} n^{a}+\omega^{-2} \Omega \nabla^{a} \omega$. Using this freedom, it is always possible to consider a conformal completion so that $\nabla_{a} n^{a}=0$ on $I$. This gauge-fixing will be preserved under conformal gauge transformations as long as we restrict consideration to functions $\omega$ that satisfy $n^{a} \nabla_{a} \omega=\mathcal{L}_{n} \omega=0$ on $I$. This gauge-fixing condition, together with property (c) above and the formula for the Ricci tensor under conformal transformations, implies $\nabla_{a} n_{b}=0$ on $I$, or equivalently $\nabla_{a} n_{b}=\nabla_{(a} n_{b)}=\frac{1}{2} \mathcal{L}_{n} g_{a b}=0$ on I. Furthermore, suppose we have any two divergence-free conformal frames associated to $\Omega$ and $\Omega^{\prime}$. Because the relative conformal factor $\omega$ obeys $\mathcal{L}_{n} \omega=0$ on $I$, the vector field $n^{a}$ is complete if and only if $n^{\prime a}$ is complete. An asymptotically flat space-time is called asymptotically Minkowski if $I$ is complete in any divergence-free conformal frame.

Denote by $\mathcal{I}$ a diffeomorphic copy of $I$, and let $\xi: \mathcal{I} \rightarrow M$ the corresponding smooth map. The pull-back, denoted by $\xi^{*}$, is defined on all covariant tensor fields in $M$ in a natural way. It can also be extended to those contravariant tensor fields such that their contraction of each of their indices with $n_{a}$ gives zero at $I$. Set $\underline{\mathrm{n}}^{a}:=\xi^{*}\left(n^{a}\right), \mathrm{g}_{a b}:=\xi^{*}\left(g_{a b}\right)$, and $\underline{\omega}:=\xi^{*}(\omega)$. It follows from the discussion above that $\mathcal{I}$ is endowed with the following universal structure. It is homeomorphic to $\mathbb{S}^{2} \times \mathbb{R}$, and equipped with pairs of fields $\left(\mathrm{g}_{a b}, \underline{\mathrm{n}}^{c}\right)$ such that:

(i) $\mathrm{g}_{a b}$ is a degenerate metric of signature $0,+,+$ with $\mathrm{g}_{a b} \underline{\mathrm{h}}^{b}=0$ and $\mathcal{L}_{\underline{\mathrm{n}}} \mathrm{g}_{a b}=0$;

(ii) $\underline{\mathrm{n}}^{a}$ is complete; and, 
(iii) any two pairs $\left(\mathrm{g}_{a b}, \underline{\mathrm{n}}^{c}\right)$ and $\left(\mathrm{g}_{a b}^{\prime}, \underline{\underline{\prime}}^{\prime c}\right)$ in the collection are related by a conformal rescaling $\Omega \rightarrow \underline{\omega} \Omega$ as $\mathrm{g}_{a b}^{\prime}=\underline{\omega}^{2} \mathrm{~g}_{a b}$, $\underline{\mathrm{n}}^{\prime a}=\underline{\omega}^{-1} \underline{\mathrm{n}}^{a}$, with $\mathcal{L}_{n} \underline{\underline{\omega}}=0.8$

This collection exists in any asymptotically Minwkoskian spacetime, and thereby receives the name of universal structure. A choice of one element $\left(\mathrm{g}_{a b}, \underline{\mathrm{n}}^{c}\right)$ of the collection $\left\{\left(\mathrm{g}_{a b}, \underline{\mathrm{n}}^{c}\right)_{i}\right\}_{i \in I}$ will be called a choice of conformal frame. Note that, since 2-spheres carry a unique conformal structure, every $\mathrm{g}_{a b}$ in this collection is conformal to a unit 2 -sphere metric. Because of this, it is sometimes convenient to restrict the remaining conformal freedom at $I$ (i.e. to fully fix the gauge function $\omega$ ) by demanding that the metric $q_{a b}$ on these 2-spheres be the metric of the unit radius 2 -sphere. This is always possible, and this conformal frame is known as the Bondi frame.

The metric in $\left(M, g_{a b}\right)$ allows the raising and lowering of indices, introduces an alternating tensor field $\epsilon^{a b c d}$ unique up to a sign, and leads to a preferred derivative operator $\nabla_{a}$ and its associated curvature tensor $R_{a b c}{ }^{d}$. Suppose we are given a fixed conformal frame. We study now what the corresponding apparatus is for $\left(\mathcal{I}, \mathrm{g}_{a b}, \underline{\mathrm{n}}^{a}\right)$. This is not a trivial question since $g_{a b}$ is a degenerate metric. In the following we will define what fields, operations, etc one can construct from this conformal frame, and then study their behaviour under a conformal gauge transformation.

First of all, we can lower indices with $\mathrm{g}_{a b}$, but we cannot raise indices a priori since $\mathrm{g}_{a b}$ is degenerate and hence it does not have an inverse. Define a tensor $\mathrm{g}^{a b}$ by the property: $\mathrm{g}_{a m} \mathrm{~g}^{m n} \mathrm{~g}_{n b}=\mathrm{g}_{m n}$. This is unique up to addition of a tensor of the form $v^{(a} \underline{\underline{n}}^{b)}$, for any vector field $v^{a}$. We will use this $\mathbf{g}^{a b}$ to raise indices whenever the lack of uniqueness does not lead to an ambiguous result. Next, we introduce an alternating tensor field $\epsilon^{a b c}$, up to a sign, by the equation $\epsilon^{a m n} \epsilon^{b p q} \underline{\mathrm{g}}_{m p} \underline{g}_{n q}=2 \underline{\mathrm{n}}^{a} \underline{\mathrm{n}}^{b}$ and demanding antisymmetry. Having fixed the sign, we can define uniquely the tensor $\epsilon_{a b c}$ by $\epsilon^{a b c} \epsilon_{a b c}=6$ and the condition of antisymmetry. The above definition implies that $\epsilon^{a b c}=\xi^{*}\left(\epsilon^{a b c d} n_{d}\right)$, but note that $\epsilon_{a b c} \neq \epsilon^{a^{\prime} b^{\prime} c^{\prime}} \mathrm{g}_{a a^{\prime}} \mathrm{g}_{b b^{\prime}} \mathrm{g}_{c c^{\prime}}=\xi^{*}\left(\epsilon_{a b c d} n^{d}\right)=0$. The usual identities for $\epsilon_{a b c}$ and $\epsilon^{a b c}$ hold.

As commented above, the universal structure of $I$ is common to every asymptotic Minkowski spacetime. The $\mathbb{S}^{2} \times \mathbb{R}$ differentiable structure together with the collection of pairs $\left(\mathrm{g}_{a b}, \underline{\mathrm{n}}^{c}\right)$ is called the zeroth order structure of $I$, and is available in any asymptotic Minkowski spacetime. We shall describe now higher order geometrical structures that are not universal, that contain specific physical information of the given space-time. The connection $D$ defined intrinsically on $I$ in any given conformal completion, induced by the torsion-free connection $\nabla$ compatible with $g_{a b}$, will be regarded as the first order structure. As we shall see, it contains the "radiative information" of the physical space-time $\left(\tilde{M}, \tilde{g}_{a b}\right)$ and consequently it changes from one space-time to another.

We define the derivative operator in $\mathcal{I}$ by $D_{a} \mu_{b}:=\xi^{*}\left(\nabla_{a} \nu_{b}\right)$, where $\mu$ is any 1 -form in $\mathcal{I}$, and $\nu_{a}$ is a 1 -form in $M$ such that $\mu_{a}=\xi^{*}\left(\nu_{a}\right)$. This derivative operator is defined intrinsically in $\mathcal{I}$. Notice that given any $\mu_{a}$ in $\mathcal{I}$, there exists many $\nu_{a}$ in $M$ that satisfies $\mu_{a}=\xi^{*}\left(\nu_{a}\right)$. However, it can be shown that the derivative operator is a well-defined operation: given two $\nu_{a}, \nu_{a}^{\prime}$ that leads to $\mu_{a}$ in $\mathcal{I}$, one actually has $\xi^{*}\left(\nabla_{a} \nu_{b}\right)=\xi^{*}\left(\nabla_{a} \nu_{b}^{\prime}\right)$. Having seen this, we can now extend the derivative operator to all tensor fields in the usual manner. In particular, given that $\nabla_{a} g_{b c}=0$, and $\nabla_{a} n^{b}=0$ on $I$, we find $D_{a \underline{\mathrm{g}}} \mathrm{ac}_{c c}=0, D_{a} \underline{\mathrm{n}}^{b}=0$ (it is also not difficult to prove also that $D_{a} \epsilon_{b c d}=0, D_{a} \epsilon^{b c d}=0$ ). In other words, this derivative operator is compatible with the metric $\mathrm{g}_{a b}$. However, it should be remarked that this connection is not uniquely defined because $\mathrm{g}_{a b}$ is degenerate. We shall now characterize in physical terms the different allowed derivative operators.

First of all, we need to know how any derivative operator changes under a conformal gauge transformation $\Omega \rightarrow \omega \Omega$. For any covector $k_{a}$, the transformation rule, at points of $I$, is

$$
D_{a}^{\prime} k_{b}=D_{a} k_{b}-2 \underline{\omega}^{-1} k_{(a} D_{b)} \underline{\omega}+\underline{\omega}^{-1}\left(\nabla^{m} \underline{\omega}\right) k_{m} \mathbf{g}_{a b} .
$$

Notice that, even when $\underline{\omega}=1$ so that $\underline{g}_{a b}$ and $\underline{\mathrm{n}}^{a}$ are invariant, $D_{a}$ changes non-trivially as $D_{a}^{\prime} k_{b}=D_{a} k_{b}+f\left(\underline{\mathrm{n}}^{m} k_{m}\right) \mathrm{g}_{a b}$, where we introduced $\nabla^{a} \underline{\omega}=: f \underline{\mathrm{n}}^{a}$. This shows that the derivative operator at $I$ is not invariant under conformal gauge transformations (in analogy to the magnetic potential in electrodynamics). Because this residual transformation of the derivative operator is just pure gauge, one is motivated to define an equivalence class of connections $\left\{D_{a}\right\}$, in a given conformal frame $\left(\mathrm{g}_{a b}, \underline{\mathrm{n}}^{c}\right)$, by the equivalence relation:

$$
D_{a} \sim D_{a}^{\prime} \quad \text { iff } \quad\left(D_{a}^{\prime}-D_{a}\right) k_{b}=\left(f \underline{\underline{n}}^{c} k_{c}\right) \underline{g}_{a b},
$$

where $f$ is an arbitrary function on $\mathcal{I}$. Now, given two connections $D_{a}, D_{a}^{\prime}$ belonging to different equivalence classes, their difference when acting on any covector is linear, and thus it must be determined by another tensor $C_{a b}{ }^{c}$ : $\left(D_{a}-D_{a}^{\prime}\right) k_{b}=C_{a b}{ }^{c} k_{c}$. The torsion-free derivative $\nabla_{a}$ implies that $C_{a b}{ }^{c}=C_{(a b)}{ }^{c}$ (just take $k_{b}=D_{b} g$ in the previous equation for some function $g$ to find $C_{a b}{ }^{c}=C_{b a}{ }^{c}$ ). On the other hand, the condition $D_{a} \underline{\underline{n}}^{b}=D_{a}^{\prime} \underline{\mathrm{n}}^{b}=0$ implies $C_{a b} \underline{\mathrm{n}}^{b}=0$, and the metric compatibility $D_{a} \mathrm{~g}_{b c}=D_{a}^{\prime} \mathrm{g}_{b c}=0$ implies $C_{a(b}{ }_{\mathrm{g}_{c) d}}=0$. Since the only vector that anihilates the metric is $\underline{\mathrm{n}}^{d}$, then

$$
\left(D_{a}-D_{a}^{\prime}\right) k_{b}=\Sigma_{a b} \underline{\underline{\mathrm{n}}}^{c} k_{c}
$$

\footnotetext{
8 The result $\xi^{*}\left(n_{a}\right)=D_{a} \xi^{*}(\Omega)=0$ implies $\underline{\mathrm{g}}_{a b} \underline{\mathrm{n}^{b}}=0$. On the other hand, because the pull-back conmutes with the Lie derivative, we automatically inherit $\mathcal{L}_{\underline{\mathrm{n}}} \underline{\mathrm{g}}_{a b}=0$ and $\underline{\mathcal{L}_{\underline{\mathrm{n}}}} \underline{\underline{\omega}}=0$.
} 
for some tensor $\Sigma_{a b}$ with $\Sigma_{a b}=\Sigma_{(a b)}$ and $\Sigma_{a b} \underline{\underline{\underline{n}}}^{b}=0$. Consequently, due to (B2) the difference $\left\{D_{a}^{\prime}\right\}-\left\{D_{a}\right\}$ between the equivalence classes of connections is fully characterized by the trace-free tensor: $\sigma_{a b}:=\Sigma_{a b}-\frac{1}{2} \Sigma_{c d} \mathrm{~g}^{c d} \mathrm{~g}_{a b}$. The space of equivalence classes $\left\{D_{a}\right\}$ is an affine space, we can select any $\left\{D_{a}^{0}\right\}$ as an origin, and then any other $\left\{D_{a}\right\}$ is labeled uniquely by a transverse $\left(\sigma_{a b} \underline{\mathrm{n}}^{b}=0\right)$ trace-free symmetric tensor $\sigma_{a b}$ on $\mathcal{J}^{+}$. These properties allow to write $\sigma_{a b}=\sigma m_{a} m_{b}+$ c.c., for some complex function $\sigma$. In physical terms, the two independent components of $\sigma_{a b}$ represent the two radiative degrees of freedom of the gravitational field in full general relativity.

We turn now to study the second order structure of an asymptotically Minkowski spacetime. Let $R_{a b c}{ }^{d}$ be the Riemann tensor of the unphysical spacetime, defined by $\nabla_{[a} \nabla_{b]} k_{c}=\frac{1}{2} R_{a b c}{ }^{d} k_{d}$ for any covector $k_{c}$. The Riemann tensor can be splitted into a totally traceless part (the Weyl tensor $C_{a b c}{ }^{d}$ ) plus a trace-full part (the Ricci $R_{a b}$, or alternatively, Schouten tensor $\left.S_{a b}\right)$ as $R_{a b c d}=C_{a b c d}+g_{a[c} S_{d] b}-g_{b[c} S_{d] a}$. It is a fundamental result [31] that the Weyl tensor vanishes at $I$, and consequently all the information about the curvature of $I$ will be determined by $S_{b}^{a}$. On the other hand, let us introduce the combination $L_{a}^{b}:=\hat{R}_{a}^{b}-\frac{1}{6} \hat{R} \delta_{b}^{a}$ and $L_{a b}=g_{a c} L_{b}^{c}$, where $\hat{R}_{a}^{b}$ and $\hat{R}$ denote the Ricci tensor and scalar curvature of the unphysical spacetime. From the standard formula for he behaviour of the Ricci tensor under conformal transformations, and using property (c) above, one can find:9

$$
\Omega S_{a b}+2 \nabla_{a} n_{b}-\Omega^{-1} n^{c} n_{c} g_{a b}=\Omega^{-1} L_{a b}=O\left(\Omega^{3}\right) .
$$

From this equation one deduces that, at points of $I, 2 \nabla_{a} n_{b}=2 \nabla_{(a} n_{b)}=\mathcal{L}_{n} g_{a b}=\Omega^{-1} n^{c} n_{c} g_{a b}$. But remember that $\mathcal{L}_{n} g_{a b}=0$ at points of $I$, so $f:=\Omega^{-1} n^{c} n_{c}=0$ at points of $I$ too. Now, contracting the above equation with $n^{b}$ and rearranging terms, one arrives at

$$
S_{a b} n^{b}+\nabla_{a} f=O\left(\Omega^{2}\right) .
$$

Since $f$ vanishes at $I$, it serves to define this hypersurface, and so its gradient must be transverse to it. Since the only trasnverse covector to $I$ is $n_{a}$, we necessarily have $\nabla_{a} f \propto n_{a}$. Then, $S_{a}^{b} n_{b} \propto n_{a}$, and vanishes at $I$. This means that the pull-back is well-defined on the tensor $S_{a}^{b}$, so we define $\underline{\mathrm{S}}_{a}^{b}:=\xi^{*}\left(S_{a}^{b}\right)$ and also $\underline{\mathrm{S}}_{a b}:=\mathrm{g}_{a c} \underline{\mathrm{S}}_{b}^{c}$. Notice the properties $\underline{\mathrm{S}}_{a b} \underline{\mathrm{n}}^{b}=0$ and $\underline{\mathrm{S}}_{a b}=\underline{\mathrm{S}}_{(a b)}$. There is one further property of $S_{a b}$ that is important to keep in mind. By taking the pull-back of the Riemann tensor, and recalling the vanishing of $C_{a b c d}$ at $I$, one gets

$$
\underline{\mathrm{R}}_{a b c}{ }^{d}=\mathrm{g}_{c[a} \underline{\mathrm{S}}_{b]}^{d}+\underline{\mathrm{S}}_{c[a} \underline{\delta}_{b]}^{d} .
$$

If we define $\underline{\mathrm{R}}_{a b c d}:=\underline{\mathrm{R}}_{a b c}{ }^{e} \mathrm{~g}_{d e}$, then the contraction of any of its indices with $\underline{\mathrm{n}}^{a}$ is zero. The corresponding Ricci tensor $R_{a b}:=\mathrm{g}^{c d} \underline{\mathrm{R}}_{a b c d}$ and scalar curvature $\underline{\mathrm{R}}:=\mathrm{g}^{c d} R_{c d}$ are thus unambiguous. Since $\underline{\mathrm{R}}_{a b c d}$ lives in the 2 dimensions orthogonal to $\underline{\mathrm{n}}^{a}$, it can be reconstructed from its scalar curvature alone, $\underline{\mathrm{R}}_{a b c d}=\underline{\operatorname{Rg}}_{a[c} \underline{g}_{d] b}$. Combining this with (B6), one finally gets $\mathrm{g}^{a b} \underline{\mathrm{S}}_{a b}=\underline{\mathrm{R}}$.

The tensor $\underline{\mathrm{S}}_{a b}$ carries information of major importance about gravitational radiation in the given spacetime, but there is still a small complication. If we change the conformal frame, this tensor transforms in a complicated way:

$$
\underline{\mathrm{S}}_{a b}^{\prime}=\underline{\mathrm{S}}_{a b}-2 \underline{\omega}^{-1} D_{a} D_{b} \underline{\omega}+4 \underline{\omega}^{-2} D_{a} \underline{\omega} D_{b} \underline{\omega}-\underline{\omega}^{-2}\left(\mathrm{~g}^{m n} D_{m} \underline{\omega} D_{n} \underline{\omega}\right) \mathrm{g}_{a b} \text {. }
$$

Consequently, a portion of this curvature is "gauge" in the sense that it contains information that is not truly physical. The goal is to extract information from this curvature tensor that remains invariant under conformal gauge transformations. This was succesfully done in [31]: given any conformal frame $\left(\mathrm{g}_{a b}, \underline{\mathrm{n}}^{c}\right)$, it can be proven that there exists a unique tensor field $\rho_{a b}$ on $I$ that fulfills:

$$
\rho_{[a b]}=0, \quad \rho_{a b} \underline{\underline{n}}^{b}=0, \quad \rho_{a b} \mathrm{~g}^{a b}=\underline{\mathrm{R}}, \quad D_{[a} \rho_{b] c}=0,
$$

and, most importantly, transforms exactly as $\underline{\mathrm{S}}_{a b}$ does under a conformal gauge reescaling. Therefore, the combination

$$
N_{a b}:=\underline{\mathrm{S}}_{a b}-\rho_{a b},
$$

is conformally gauge invariant. Consequently, the role of $\rho_{a b}$ is to subtract from $S_{a b}$ the pure gauge-dependent contribution. In a Bondi conformal frame, in particular, one has $\rho_{a b}=\frac{1}{2} \underline{\mathrm{g}}_{a b} \underline{\mathrm{R}}$. $N_{a b}$ is referred to as the Bondi news tensor and is regarded as the second order structure at $\mathcal{J}$. It satisfies

$$
N_{[a b]}=0, \quad N_{a b} \underline{\mathrm{n}}^{b}=0, \quad N_{a b \underline{\mathrm{g}}^{a b}}=0 .
$$

This is all the physical information we can extract from $S_{a b}$. Nevertheless, the full information of the curvature of $\left\{D_{a}\right\}$ is actually contained in $\underline{\mathrm{S}}_{b}^{a}$, and not in $\underline{\mathrm{S}}_{a b}$ (notice that since $\mathrm{g}_{a b}$ is not invertible it is not possible to reconstruct

\footnotetext{
9 The definition of asymptotically Minkowski spacetimes requires that $\hat{T}_{a b}=O\left(\Omega^{2}\right)$, and so $\hat{R}_{a b}=O\left(\Omega^{2}\right)$. Then $L_{a b}=g_{b c} L_{a}^{c}=O\left(\Omega^{4}\right)$.
} 
$\underline{\mathrm{S}}_{b}^{a}$ from $\underline{\mathrm{S}}_{a b}$.). This information is encoded in what we shall call the third (and last) geometric asymptotic structure, which can be worked out from the Weyl tensor. Since the Weyl tensor vanishes at $I$, the tensor $\Omega^{-1} C_{a b c d}$ is smooth up to and including $I$. If we define:

$$
\begin{aligned}
K^{a b} & :=\epsilon^{a m n} \epsilon^{b p q} \xi^{*}\left(\Omega^{-1} C_{m n p q}\right), \\
{ }^{*} K^{a b} & :=\epsilon^{a m n} \epsilon^{b p q} \xi^{*}\left(\Omega^{-1 *} C_{m n p q}\right),
\end{aligned}
$$

then we immediately see that they are symmetric and that $K^{a b} \mathrm{~g}_{a b}={ }^{*} K^{a b} \mathrm{~g}_{a b}=0$. Taking the curl of (B) $)$, using the definition of Riemann tensor, expressing it in terms of the Weyl tensor, and doing some manipulations, it is possible to show that $D_{[a} \underline{S}_{b]}^{c}=\frac{1}{4} \epsilon_{a m n}{ }^{*} K^{m c}$, which automatically leads to

$$
D_{[a} N_{b] c}=\frac{1}{4} \epsilon_{a m n}{ }^{*} K^{m n} \mathrm{~g}_{n c},
$$

or, equivalently, ${ }^{*} K^{a b}=2 \epsilon^{p q a} D_{p} N_{q}{ }^{b}$. Furthermore, a straightforward calculation shows that ${ }^{*} K^{a b}$ remains invariant under conformal gauge transformations with $\omega=1$, so it is a physically meaningful quantity. Because ${ }^{*} K^{a b}$ involves derivatives of $S_{a}^{b}$, it is called the third order structure at $I$.

If ${ }^{*} K^{a b}=0$, then $N_{a b}=0$, and the associated equivalence class $\left\{D_{a}\right\}$ of connections is said to be trivial. In this case, the physical space-time $\left(\hat{M}, \hat{g}_{a b}\right)$ does not contain gravitational radiation. In particular, every stationary, asymptotically flat spacetime produce a trivial connection on $I$. Conversely, if $N_{a b}=0$ (i.e. no gravitational waves), it can be shown that the spacetime is stationary [45].

\section{Appendix C: Spin-coefficient formalism and asymptotic behaviour}

Let $\left(M, g_{a b}\right)$ be a spacetime and $\left\{\ell^{a}, n^{a}, m^{a}, \bar{m}^{a}\right\}$ a Newman-Penrose basis, i.e. a null tetrad satisfying $n^{a} \ell_{a}=1$, $m^{a} \bar{m}_{a}=-1$, and zero otherwise 10 If we introduce the notation $e_{1}^{a}=\ell^{a}, e_{2}^{a}=n^{a}, e_{3}^{a}=m^{a}, e_{4}^{a}=\bar{m}^{a}$, then this basis of null vectors satisfies $g_{a b}=\eta_{i j} e_{a}^{i} e_{b}^{j}$ with $\eta_{12}=\eta_{21}=1, \eta_{34}=\eta_{43}=-1$. Internal indices $(i, j, \ldots)$ are raised and lowered with $\eta_{i j}$, while spacetime indices $(a, b, \ldots)$ are raised and lowered with $g_{a b}$.

Given this tetrad we can introduce the connection 1-form by $\gamma_{c}{ }^{b}{ }_{a}:=-e_{c}^{i} \nabla_{a} e_{i}^{b}$, which satisfies $\gamma_{a b c}=-\gamma_{b a c}$. In this basis there are 12 independent (complex) components of the connection 1-form, which are called spin coefficients. They are designated by

$$
\begin{array}{ccc}
\kappa=\gamma_{311}=-m^{a} \ell^{b} \nabla_{b} \ell_{a}, & \rho=\gamma_{314}=-m^{a} \bar{m}^{b} \nabla_{b} \ell_{a}, & \epsilon=\frac{1}{2}\left(\gamma_{211}+\gamma_{341}\right)=-\frac{1}{2}\left(n^{a} \ell^{b} \nabla_{b} \ell_{a}-\bar{m}^{a} \ell^{b} \nabla_{b} m_{a}\right), \\
\sigma=\gamma_{313}=-m^{a} m^{b} \nabla_{b} \ell_{a}, & \mu=\gamma_{243}=\bar{m}^{a} m^{b} \nabla_{b} n_{a}, & \gamma=\frac{1}{2}\left(\gamma_{212}+\gamma_{342}\right)=-\frac{1}{2}\left(n^{a} n^{b} \nabla_{b} \ell_{a}-\bar{m}^{a} n^{b} \nabla_{b} m_{a}\right), \\
\lambda=\gamma_{244}=\bar{m}^{a} \bar{m}^{b} \nabla_{b} n_{a}, & \tau=\gamma_{312}=-m^{a} n^{b} \nabla_{b} \ell_{a}, & \alpha=\frac{1}{2}\left(\gamma_{214}+\gamma_{344}\right)=-\frac{1}{2}\left(n^{a} \bar{m}^{b} \nabla_{b} \ell_{a}-\bar{m}^{a} \bar{m}^{b} \nabla_{b} m_{a}\right), \\
\nu=\gamma_{242}=\bar{m}^{a} n^{b} \nabla_{b} n_{a}, & \pi=\gamma_{241}=\bar{m}^{a} \ell^{b} \nabla_{b} n_{a}, & \beta=\frac{1}{2}\left(\gamma_{213}+\gamma_{343}\right)=-\frac{1}{2}\left(n^{a} m^{b} \nabla_{b} \ell_{a}-\bar{m}^{a} m^{b} \nabla_{b} m_{a}\right) .
\end{array}
$$

Note that $\gamma_{311}=\bar{\gamma}_{411}, \gamma_{314}=\bar{\gamma}_{413}$, etc. On the other hand, the Weyl tensor has ten independent components which are represented in this framework by 5 complex scalars:

$$
\begin{aligned}
& \Psi_{0}:=-C_{1313}=-C_{a b c d} \ell^{a} m^{b} \ell^{c} m^{d}, \\
& \Psi_{1}:=-C_{1213}=-C_{a b c d} \ell^{a} n^{b} \ell^{c} m^{d}, \\
& \Psi_{2}:=-C_{1342}=-C_{a b c d} \ell^{a} m^{b} \bar{m}^{c} n^{d}, \\
& \Psi_{3}:=-C_{1242}=-C_{a b c d} \ell^{a} n^{b} \bar{m}^{c} n^{d}, \\
& \Psi_{4}:=-C_{2424}=-C_{a b c d} n^{a} \bar{m}^{b} n^{c} \bar{m}^{d} .
\end{aligned}
$$

The remaining components are determined using the symmetry properties of the Weyl tensor. In particular, it is not difficult to show that

$$
\Psi_{1}=C_{1334}=C_{1231}
$$

${ }^{10}$ In this appendix and in the next one we follow the Newman-Penrose [34] notation. In particular, the metric signature will be $(+,-,-,-)$ in order to use the asymptotic expressions for the spin-coefficients calculated in [46]. 


$$
\begin{aligned}
\Psi_{3} & =C_{2443}, \\
\operatorname{Re} \Psi_{2} & =\frac{-1}{2} C_{1212}=\frac{-1}{2} C_{3434}, \\
\operatorname{Im} \Psi_{2} & =\frac{1}{2 i} C_{1234}, \\
C_{1314} & =C_{2324}=C_{1332}=C_{1442}=0 .
\end{aligned}
$$

In asymptotically flat spacetimes, a preferred coordinate system and an associated null tetrad can always be considered. Following Bondi and Sachs, we can always introduce a foliation of the asymptotic region of $M$ by outgoing null hypersurfaces $\{u=$ const $\}$. Denoting the corresponding geodesic null normal by $\ell^{a}$, we can introduce an affine parameter $r$ of $\ell^{a}$ (i.e. $\ell=\frac{\partial}{\partial r}$ so that $\ell^{a} \nabla_{a} r=1$ ) such that each null surface $u=$ const is foliated by a family of (space-like) 2 -spheres $\{r=$ const $\}$. The set $\{u, r, \theta, \phi\}$ is called Bondi-Sachs coordinates. Let us denote the intrinsic $(-,-)$ metric of these 2 -spheres by $q_{a b}$ and the other null-normal to each of these 2 -spheres by $n^{a}$, normalized so that $g_{a b} \ell^{a} n^{b}=1$. If $\ell^{a}$ is normal to the $\{u=$ const $\}$ hypersurfaces, necessarily $\ell^{a}=g^{a b} \nabla_{b} u$, so that $\ell_{a}:=g_{a b} \ell^{b}=\nabla_{a} u$ and we can write $n=V \frac{\partial}{\partial u}+U \frac{\partial}{\partial r}+X^{A} \frac{\partial}{\partial X^{A}}$ with $V=1\left(n_{a}\right.$ is not simply given by $\nabla_{a} r$ since $n^{a}$ is not normal to $\{r=$ const $\}$ hypersurfaces in general). Finally, introduce a null complex vector field $m^{a}$ and its complex conjugate $\bar{m}^{a}$ such that their real and imaginary parts are tangential to these 2 -spheres, and they are normalized such that $g_{a b} m^{a} \bar{m}^{b}=-1$. Thus, at each point in the asymptotic region we have a null tetrad $\left\{\ell^{a}, n^{a}, m^{a}, \bar{m}^{a}\right\}$ for which the only non-zero contractions are $\ell \cdot n=1$ and $m \cdot \bar{m}=-1$. In terms of the null tetrad, the metric takes the form $g_{a b}=2 n_{(a} \ell_{b)}-2 m_{(a} \bar{m}_{b)}$.

The spin-coefficient formalism is particularly useful for asymptotically flat spacetimes. If the Weyl scalars are smooth functions on the spacetime manifold, their asymptotic behaviour as $r \rightarrow \infty$, keeping $u, \theta, \phi$ constant, is determined by the Peeling theorem [36]:

$$
\Psi_{i}(u, r, \theta, \phi) \sim \Psi_{i}^{0}(u, \theta, \phi) / r^{5-i}, \quad i=0,1,2,3,4 .
$$

Furthermore, the asymptotic behaviour of the spin-coefficients can be systematically obtained by integrating asymptotically a set of equations in the Newman-Penrose framework that are equivalent to Einstein's field equations [46]. The results read

$$
\begin{aligned}
& \lambda=\lambda^{0} / r+O\left(r^{-2}\right), \quad \lambda^{0}=\dot{\bar{\sigma}}^{0}, \\
& \mu=\mu^{0} / r+O\left(r^{-2}\right), \quad \mu^{0}=-1, \\
& \sigma=\sigma^{0} / r^{2}+O\left(r^{-4}\right), \quad \sigma^{0}=\text { free data }, \\
& \rho=\rho^{0} / r+\rho^{1} / r^{3}+O\left(r^{-5}\right), \quad \rho^{0}=-1, \quad \rho^{1}=-\left|\sigma^{0}\right|^{2}, \\
& \kappa=0, \\
& \pi=0, \\
& \nu=\nu^{0}+O\left(r^{-1}\right), \quad \nu^{0}=0, \\
& \tau=\bar{\alpha}+\beta=\left(\bar{\alpha}^{0}+\beta^{0}\right) / r+O\left(r^{-2}\right), \quad\left(\bar{\alpha}^{0}+\beta^{0}\right)=0 .
\end{aligned}
$$

and $\Psi_{4}^{0}=-\ddot{\bar{\sigma}}^{0}$.

The relation with the Bondi News $N_{33}=N_{a b} m^{a} m^{b}$ introduced in Appendix A can be obtained using (B12) and the result ${ }^{*} K^{a b}=2 \epsilon^{p q a} D_{p} N_{q}{ }^{b}$. Using the first equation we get ${ }^{*} K^{a b} m_{a} m_{b}=4 i n^{a} m^{b} n^{c} m^{d} \xi^{*}\left(\Omega^{-1} C_{a b c d}\right)=-4 i \bar{\Psi}_{4}^{0}$; on the other hand, the second equation yields ${ }^{*} K^{a b} m_{a} m_{b}=2 i n^{p} D_{p}\left(N_{a b} m^{a} m^{b}\right)=2 i \partial_{u} N_{33}$. Combining both we get $\dot{N}_{33}=-2 \bar{\Psi}_{4}^{0}=2 \ddot{\sigma}^{0}$. Furthermore, from the Bondi mass formula $\int_{\mathcal{J}} d u d \mathbb{S}^{2}\left|N_{33}\right|^{2}<\infty$ one infers $N_{33} \rightarrow 0$ at $u \rightarrow \pm \infty$ so $N_{33}=2 \dot{\sigma}^{0}$.

\section{Appendix D: Alternative derivation using the Spin-Coefficient formalism}

In this appendix we derive the result (51) using the spin-coefficient formalism and the corresponding asymptotic behaviour summarized in the previous appendix. Our starting point is equation (42), which in the physical spacetime $\left(\hat{M}, \hat{g}_{a b}\right)$ can be rewritten in a similar way (in this appendix we only work with the physical spacetime so we will omit the hat symbol in all associated geometric quantities for convenience)

$$
\int_{\hat{M}} p_{1}(\hat{R})=-\lim _{r_{0} \rightarrow \infty} \frac{1}{8 \pi^{2}} \int_{r=r_{0}}\left(K_{a d}-K^{\prime}{ }_{a d}\right) \hat{n}^{e} R_{b c}{ }^{d} \epsilon^{a b c f} \hat{n}_{f} \sqrt{h} d^{3} x
$$


where here $\hat{n}_{b}=\frac{1}{\sqrt{g^{a b} \nabla_{a} r \nabla_{b} r}} \nabla_{b} r$ is the normal vector to $\left\{r=r_{0}\right\}$ hypersurfaces and $K_{a b}=D_{a} \hat{n}_{b}$ the extrinsic curvature. In terms of the Newman-Penrose basis constructed in Appendix C we must have $\nabla_{a} r=a_{1} n_{a}+a_{2} \ell_{a}$. Given that $\ell^{a} \nabla_{a} r=1$, then $\nabla_{a} r=n_{a}+a_{2} \ell_{a}$; and squaring $n_{a}=\nabla_{a} r-a_{2} \ell_{a}$ we get $a_{2}=\frac{1}{2} g^{r r}$ so that $\nabla_{a} r=$ $n_{a}+\frac{g^{r r}}{2} \ell_{a}$. The asymptotic behaviour of $g^{r r}$ in Bondi-Sachs coordinates can be found in [37, 38], and is given by $-g^{r r}=1-\frac{2 M}{r}+O\left(r^{-2}\right)$. Note that $D_{a} \hat{n}_{b}=h_{a}^{a^{\prime}} h_{b}^{b^{\prime}} \nabla_{b} n_{b^{\prime}}=h_{a}^{a^{\prime}} \nabla_{a} \hat{n}_{b}$ and $\epsilon^{a b c h} \hat{n}_{h} D_{a} \hat{n}_{e}=\epsilon^{a b c h} \hat{n}_{h} \nabla_{a} \hat{n}_{e}$. We can also take the prefactor of $\hat{n}_{b}$ out of the derivative operator thanks to the antisymmetry of the Riemann tensor, $\left(\nabla_{a} \hat{n}_{e}\right) \hat{n}_{d} R_{b c}{ }^{d e}=\nabla_{a}\left[\left(g^{r r}\right)^{-1 / 2} \nabla_{e} r\right] \hat{n}_{d} R_{b c}{ }^{d e}=\left(g^{r r}\right)^{-1 / 2} \nabla_{a}\left[\nabla_{e} r\right] \hat{n}_{d} R_{b c}{ }^{d e}+0$. Finally, if we take the $\left\{r=r_{0}\right\}$ surface outside the gravitational sources (we assume they have spatial compact support), then $R_{a b c d}=C_{a b c d}$. Taking into account all this:

$$
\int_{\hat{M}} p_{1}(\hat{R})=\lim _{r_{0} \rightarrow \infty} \frac{1}{8 \pi^{2}} \int_{r=r_{0}} \frac{\sqrt{h}}{\left(g^{r r}\right)^{3 / 2}} d^{3} x C_{b c}{ }^{d e} \epsilon^{a b c h}\left(n_{h}+\frac{g^{r r}}{2} l_{h}\right)\left(n_{d}+\frac{g^{r r}}{2} l_{d}\right) \nabla_{a}\left(n_{e}+\frac{g^{r r}}{2} l_{e}\right) .
$$

We have $\nabla_{a}\left(n_{e}+\frac{g^{r r}}{2} l_{e}\right)=-\left(\gamma_{2 e a}+\frac{g^{r r}}{2} \gamma_{1 e a}\right)+\frac{1}{2} l_{e} \nabla_{a} g^{r r}$. Then, using $\epsilon^{a b c d}=4 ! i l^{[a} n^{b} m^{c} \bar{m}^{d]}$, we find

$\frac{-1}{8 \pi^{2}} \int \frac{6 \sqrt{h} d^{3} x}{\left(g^{r r}\right)^{3 / 2}}\left[\left(C_{2 e b c}+\frac{g^{r r}}{2} C_{1 e b c}\right) i\left(n^{[a} m^{b} \bar{m}^{c]}-\frac{g^{r r}}{2} l^{[a} m^{b} \bar{m}^{c]}\right)\left(\gamma_{2}{ }_{a}^{e}+\frac{g^{r r}}{2} \gamma_{1}^{e}{ }_{a}\right)-C_{21 b c} i\left(n^{[a} m^{b} \bar{m}^{c]}-\frac{g^{r r}}{2} l^{[a} m^{b} \bar{m}^{c]}\right) \frac{\nabla_{a} g_{\mathrm{P}}^{r r}}{2}\right]$

We do the calculation term by term:

(A)

$$
\begin{aligned}
C_{2 e b c} n n^{[a} m^{b} \bar{m}^{c]} & =\frac{1}{3} C_{2 e b c}\left[n^{a} m^{[b} \bar{m}^{c]}+m^{a} \bar{m}^{[b} n^{c]}+\bar{m}^{a} n^{[b} m^{c]}\right] \\
& =\frac{1}{3}\left[n^{a} C_{2 e 34}+m^{a} C_{2 e 42}+\bar{m}^{a} C_{2 e 23}\right]=\frac{1}{3}\left[n^{a} C_{2 e 34}+2 i \operatorname{Im}\left(m^{a} C_{2 e 42}\right)\right],
\end{aligned}
$$

(B): same as (A) but changing $n^{a} \rightarrow l^{a}$

$$
\begin{aligned}
C_{2 e b c} l^{[a} m^{b} \bar{m}^{c]} & =\frac{1}{3} C_{2 e b c}\left[l^{a} m^{[b} \bar{m}^{c]}+m^{a} \bar{m}^{[b} l^{c]}+\bar{m}^{a} l^{[b} m^{c]}\right] \\
& =\frac{1}{3}\left[l^{a} C_{2 e 34}+m^{a} C_{2 e 41}+\bar{m}^{a} C_{2 e 13}\right]=\frac{1}{3}\left[l^{a} C_{2 e 34}+2 i \operatorname{Im}\left(m^{a} C_{2 e 41}\right)\right],
\end{aligned}
$$

(C): same as (A) but changing $C_{2 \ldots} \rightarrow C_{1 \ldots}$

$$
\begin{aligned}
C_{1 e b c} n^{[a} m^{b} \bar{m}^{c]} & =\frac{1}{3} C_{1 e b c}\left[n^{a} m^{[b} \bar{m}^{c]}+m^{a} \bar{m}^{[b} n^{c]}+\bar{m}^{a} n^{[b} m^{c]}\right] \\
& =\frac{1}{3}\left[n^{a} C_{1 e 34}+m^{a} C_{1 e 42}+\bar{m}^{a} C_{1 e 23}\right]=\frac{1}{3}\left[n^{a} C_{1 e 34}+2 i \operatorname{Im}\left(m^{a} C_{1 e 42}\right)\right],
\end{aligned}
$$

(D): same as (B) but changing $C_{2 \ldots} \rightarrow C_{1 \ldots}$

$$
\begin{aligned}
C_{1 e b c} l^{[a} m^{b} \bar{m}^{c]} & =\frac{1}{3} C_{1 e b c}\left[l^{a} m^{[b} \bar{m}^{c]}+m^{a} \bar{m}^{[b} l^{c]}+\bar{m}^{a} l^{[b} m^{c]}\right] \\
& =\frac{1}{3}\left[l^{a} C_{1 e 34}+m^{a} C_{1 e 41}+\bar{m}^{a} C_{1 e 13}\right]=\frac{1}{3}\left[l^{a} C_{1 e 34}+2 i \operatorname{Im}\left(m^{a} C_{1 e 41}\right)\right],
\end{aligned}
$$

(E): same as (A) but changing $C_{2 e \ldots} \rightarrow C_{21 \ldots}$

$$
C_{21 b c} n^{[a} m^{b} \bar{m}^{c]}=\frac{1}{3}\left[n^{a} C_{2134}+2 i \operatorname{Im}\left(m^{a} C_{2142}\right)\right]=\frac{1}{3}\left[-2 i n^{a} \operatorname{Im} \Psi_{2}+2 i \operatorname{Im}\left(m^{a} \Psi_{3}\right)\right],
$$

(F): same as (B) but changing $C_{2 e \ldots} \rightarrow C_{21 \ldots}$

$$
C_{21 b c} l^{[a} m^{b} \bar{m}^{c]}=\frac{1}{3}\left[l^{a} C_{2134}+2 i \operatorname{Im}\left(m^{a} C_{2141}\right)\right]=\frac{1}{3}\left[-2 i l^{a} \operatorname{Im} \Psi_{2}-2 i \operatorname{Im}\left(m^{a} \bar{\Psi}_{1}\right)\right] .
$$

We now elaborate in detail each of these terms:

(A)

$$
\begin{aligned}
& \frac{1}{3}\left[n^{a} C_{2 e 34}+2 i \operatorname{Im}\left(m^{a} C_{2 e 42}\right)\right]\left(\gamma_{2}{ }_{a}^{e}+\frac{g^{r r}}{2} \gamma_{1}{ }_{a}^{e}\right)=\frac{1}{3}\left[\gamma_{2}{ }_{2}^{e} C_{2 e 34}+2 i \operatorname{Im}\left(\gamma_{2}{ }_{3} C_{2 e 42}\right)+\frac{g^{r r}}{2} \gamma_{1}{ }_{2}^{e} C_{2 e 34}+g^{r r} i \operatorname{Im}\left(\gamma_{1}{ }_{3}^{e} C_{2 e 42}\right)\right] \\
& =\frac{1}{3}\left[2 i \operatorname{Im}\left(\gamma_{2}{ }_{2}^{3} C_{2334}\right)+2 i \operatorname{Im}\left(\gamma_{2}{ }_{3}{ }_{3} C_{2342}+\gamma_{2}{ }_{3}^{4} C_{2442}\right)\right.
\end{aligned}
$$




$$
\begin{aligned}
& \left.+\frac{g^{r r}}{2}\left(\gamma_{1}{ }_{2}{ }_{2} C_{2134}+2 i \operatorname{Im}\left(\gamma_{1}{ }_{2}{ }_{2} C_{2334}\right)\right)+g^{r r} i \operatorname{Im}\left(\gamma_{1}{ }_{3} C_{2142}+\gamma_{1}{ }_{3} C_{2342}+\gamma_{1}{ }_{3} C_{2442}\right)\right] \\
= & \frac{1}{3}\left[-2 i \operatorname{Im}\left(\nu \bar{\Psi}_{3}\right)-2 i \operatorname{Im}\left(\bar{\lambda} \Psi_{4}\right)+2 i \operatorname{Re} \gamma \operatorname{Im} \Psi_{2} g^{r r}+i g^{r r} \operatorname{Im}\left(\bar{\tau} \bar{\Psi}_{3}\right)-2 i g^{r r} \operatorname{Re} \beta \operatorname{Im}\left(\Psi_{3}\right)+g^{r r} i \operatorname{Im}\left(\sigma \Psi_{4}\right)\right],
\end{aligned}
$$

(C): similar to $(\mathrm{A})$ changing $C_{2 \ldots} \rightarrow C_{1} \ldots$

$$
\begin{aligned}
& \frac{1}{3}\left[n^{a} C_{1 e 34}+2 i \operatorname{Im}\left(m^{a} C_{1 e 42}\right)\right]\left(\gamma_{2}{ }_{a}^{e}+\frac{g^{r r}}{2} \gamma_{1}{ }_{a}{ }_{a}\right)=\frac{1}{3}\left[\gamma_{2}{ }_{2}^{e} C_{1 e 34}+2 i \operatorname{Im}\left(\gamma_{2}{ }_{3}^{e} C_{1 e 42}\right)+\frac{g^{r r}}{2} \gamma_{1}{ }_{2} C_{1 e 34}+g^{r r} i \operatorname{Im}\left(\gamma_{1}{ }_{3} C_{1 e 42}\right)\right] \\
= & \frac{1}{3}\left[\gamma_{2}{ }_{2}{ }_{2} C_{1234}+2 i \operatorname{Im}\left(\gamma_{2}{ }^{3}{ }_{2} C_{1334}\right)+2 i \operatorname{Im}\left(\gamma_{2}{ }_{3}{ }_{3} C_{1242}+\gamma_{2}{ }_{3} C_{1342}+\gamma_{2}{ }_{3}{ }_{3} C_{1442}\right)\right. \\
& \left.+\frac{g^{r r}}{2} 2 i \operatorname{Im}\left(\gamma_{1}{ }_{2}{ }_{2} C_{1334}\right)+g^{r r} i \operatorname{Im}\left(\gamma_{1}{ }_{3}{ }_{3} C_{1342}+\gamma_{1}{ }_{3} C_{1442}\right)\right] \\
= & \frac{1}{3}\left[4 i \operatorname{Re} \gamma \operatorname{Im} \Psi_{2}-2 i \operatorname{Im}\left(\nu \Psi_{1}\right)-4 i \operatorname{Re} \beta \operatorname{Im} \Psi_{3}+2 i \operatorname{Im}\left(\mu \Psi_{2}\right)+i g^{r r} \operatorname{Im}\left(\bar{\tau} \Psi_{1}\right)-i g^{r r} \operatorname{Im}\left(\bar{\rho} \Psi_{2}\right)\right],
\end{aligned}
$$

$$
\begin{aligned}
& \frac{1}{3}\left[l^{a} C_{2 e 34}+2 i \operatorname{Im}\left(m^{a} C_{2 e 41}\right)\right]\left(\gamma_{2}{ }_{a}^{e}+\frac{g^{r r}}{2} \gamma_{1}{ }_{a}^{e}\right)=\frac{1}{3}\left[\gamma_{2}{ }_{1}^{e} C_{2 e 34}+2 i \operatorname{Im}\left(\gamma_{2}{ }_{3}^{e} C_{2 e 41}\right)+\frac{g^{r r}}{2} \gamma_{1}{ }_{1}^{e} C_{2 e 34}+g^{r r} i \operatorname{Im}\left(\gamma_{1}{ }_{3} C_{2 e 41}\right)\right] \\
& =\frac{1}{3}\left[2 i \operatorname{Im}\left(\gamma_{2}{ }^{3}{ }_{1} C_{2334}\right)+2 i \operatorname{Im}\left(\gamma_{2}{ }_{3} C_{2341}+\gamma_{2}{ }_{3}{ }_{3} C_{2441}\right)\right. \\
& \left.+\frac{g^{r r}}{2}\left(\gamma_{1}{ }_{1} C_{2134}+2 i \operatorname{Im}\left(\gamma_{1}{ }_{1}{ }_{1} C_{2334}\right)\right)+g^{r r} i \operatorname{Im}\left(\gamma_{1}{ }_{3}{ }_{3} C_{2141}+\gamma_{1}{ }_{3} C_{2341}+\gamma_{1}{ }_{3} C_{2441}\right)\right] \\
& =\frac{1}{3}\left[-2 i \operatorname{Im}\left(\pi \bar{\Psi}_{3}\right)+2 i \operatorname{Im}\left(\mu \bar{\Psi}_{2}\right)+2 i g^{r r} \operatorname{Re} \epsilon \operatorname{Im} \Psi_{2}+i g^{r r} \operatorname{im}\left(\bar{\kappa} \bar{\Psi}_{3}\right)+2 i g^{r r} \operatorname{Re} \beta \operatorname{Im}\left(\bar{\Psi}_{1}\right)-i g^{r r} \operatorname{Im}\left(\bar{\rho} \bar{\Psi}_{2}\right)\right] \text {, } \\
& \frac{1}{3}\left[l^{a} C_{1 e 34}+2 i \operatorname{Im}\left(m^{a} C_{1 e 41}\right)\right]\left(\gamma_{2}{ }_{a}^{e}+\frac{g^{r r}}{2} \gamma_{1}{ }_{a}\right)=\frac{1}{3}\left[\gamma_{2}{ }_{1}^{e} C_{1 e 34}+2 i \operatorname{Im}\left(\gamma_{2}{ }_{3}^{e} C_{1 e 41}\right)+\frac{g^{r r}}{2} \gamma_{1}{ }_{1}^{e} C_{1 e 34}+g^{r r} i \operatorname{Im}\left(\gamma_{1}{ }_{3} C_{1 e 41}\right)\right] \\
& =\frac{1}{3}\left[\gamma_{2}{ }^{2}{ }_{1} C_{1234}+2 i \operatorname{Im}\left(\gamma_{2}{ }_{1} C_{1334}\right)+2 i \operatorname{Im}\left(\gamma_{2}{ }_{3}{ }_{3} C_{1241}+\gamma_{2}{ }_{3} C_{1341}+\gamma_{2}{ }_{3}{ }_{3} C_{1441}\right)\right. \\
& \left.+\frac{g^{r r}}{2} 2 i \operatorname{Im}\left(\gamma_{1}^{3}{ }_{1} C_{1334}\right)+g^{r r} i \operatorname{Im}\left(\gamma_{1}{ }_{3} C_{1341}+\gamma_{1}{ }_{3}^{4} C_{1441}\right)\right] \\
& =\frac{1}{3}\left[4 i \operatorname{Re} \epsilon \operatorname{Im} \Psi_{2}-2 i \operatorname{Im}\left(\pi \Psi_{1}\right)+4 i \operatorname{Re} \beta \operatorname{Im} \bar{\Psi}_{1}-2 i \operatorname{Im}\left(\bar{\lambda} \bar{\Psi}_{0}\right)+i g^{r r} \operatorname{Im}\left(\bar{\kappa} \Psi_{1}\right)+i g^{r r} \operatorname{Im}\left(\sigma \bar{\Psi}_{0}\right)\right] \text {, }
\end{aligned}
$$

(E)

$$
\frac{1}{3}\left[-2 i n^{a} \operatorname{Im} \Psi_{2}+2 i \operatorname{Im}\left(m^{a} \Psi_{3}\right)\right] \nabla_{a} g^{r r}=\frac{-2 i}{3}\left[\frac{-2 M}{r^{2}}+O\left(r^{-3}\right)\right] n^{a} \nabla_{a} r \operatorname{Im} \Psi_{2}=\frac{2 M i}{3 r}\left[1+O\left(r^{-1}\right)\right] g^{r r} \operatorname{Im} \Psi_{2},
$$

(F)

$$
\frac{1}{3}\left[-2 i n^{a} \operatorname{Im} \Psi_{2}-2 i \operatorname{Im}\left(m^{a} \bar{\Psi}_{1}\right)\right] \nabla_{a} g^{r r}=\frac{-2 i}{3}\left[\frac{-2 M}{r^{2}}+O\left(r^{-3}\right)\right] n^{a} \nabla_{a} r \operatorname{Im} \Psi_{2}=\frac{2 M i}{3 r}\left[1+O\left(r^{-1}\right)\right] g^{r r} \operatorname{Im} \Psi_{2} .
$$

We use now the asymptotic properties of the spin-coefficients (see Appendix C) in the limit to future null infinity to simplify all these quantities. Notice that $\sqrt{h} \sim r^{2}$, so all terms that decay faster than $1 / r^{2}$ vanish at $\mathcal{J}^{+}$. On the other hand, all the spin-coefficients decay at least as $1 / r$, and because all terms above are of the form spin - coefficient $\times$ Weyl - scalar, the only non-vanishing contributions are those that involve $\Psi_{4}=O\left(\frac{1}{r}\right)$. Among all of them, we have to take the one whose spin-coefficient only decays as $O\left(\frac{1}{r}\right)$, which is $\lambda$. Doing this we get

$$
\int d^{4} x \sqrt{-g}\left\langle\nabla_{a} j_{5}^{a}\right\rangle=-\frac{\hbar}{6} \int_{\hat{M}} p_{1}(\hat{R})=\frac{\hbar}{12 \pi^{2}} \int_{\mathcal{J}^{+}} d u d \mathbb{S}^{2} \operatorname{Im}\left(\ddot{\sigma}^{0} \dot{\bar{\sigma}}^{0}\right) .
$$

Recalling that $N_{33}=2 \dot{\sigma}^{0}$ (see Appendix C), we recover equation (51)

[1] L. Parker Phys. Rev. Lett. 21 (1968) 562-564. 
[2] S. W. Hawking Comm. Math. Phys. 43 no. 3, (1975) $199-220$.

[3] R. Bertlmann, Anomalies in quantum field theory. Oxford, UK: Clarendon (1996) 566 p. (International series of monographs on physics: 91), 1996.

[4] S. L. Adler Phys. Rev. 177 (1969) 2426-2438.

[5] J. S. Bell and R. Jackiw Il Nuovo Cimento A (1965-1970) 60 no. 1, (1969) 47-61.

[6] M. Nakahara, Geometry, topology and physics. Bristol, UK: Hilger (1990) 505 p. (Graduate student series in physics).

[7] M. D. Schwartz, Quantum Field Theory and the Standard Model. Cambridge University Press, 2014.

[8] N. Christ Phys. Rev. D 21 (1980) 1591.

[9] I. Agullo, A. del Rio, and J. Navarro-Salas Phys. Rev. Lett. 118 (Mar, 2017) 111301.

[10] I. Agullo, A. del Rio, and J. Navarro-Salas Int. J. Mod. Phys. D 26 no. 12, (2017) 1742001.

[11] I. Agullo, A. del Rio, and J. Navarro-Salas Phys. Rev. D 98 no. 12, (2018) 125001.

[12] I. Agulló, A. del Río, and J. Navarro-Salas Symmetry 10 no. 12, (2018) 763.

[13] J. Kiskis Phys. Rev. D 18 (1978) 3690-3694.

[14] R. Jackiw Rev. Mod. Phys. 49 (Jul, 1977) 681-706.

[15] M. A. Shifman, ed., Instantons in gauge theories. World scientific, Singapore, 1994.

[16] R. Jackiw and C. Rebbi Phys. Rev. Lett. 37 (1976) 172-175.

[17] J. Callan, Curtis G., R. Dashen, and D. J. Gross Phys. Lett. B 63 (1976) 334-340.

[18] K. M. Bitar and S.-J. Chang Phys. Rev. D 17 (Jan, 1978) 486-497.

[19] G. 't Hooft Phys. Rev. Lett. 37 (Jul, 1976) 8-11.

[20] S. Hawking Phys. Lett. A 60 no. 2, (1977) $81-83$.

[21] T. Eguchi and A. J. Hanson Gen. Rel. and Grav. 11 no. 2, (1979) 315 - 320.

[22] M. Dunajski, Solitons, instantons, and twistors. 2010.

[23] G. 't Hooft Nucl. Phys. B 315 no. 2, (1989) 517-527.

[24] R. Penrose Phys. Rev. Lett. 10 (1963) 66-68.

[25] A. Ashtekar Phys. Rev. Lett. 46 (1981) 573-576.

[26] A. Ashtekar J. Math. Phys. 22 no. 12, (1981) 2885-2895.

[27] T. Eguchi, P. B. Gilkey, and A. J. Hanson, "Gravitation, gauge theories and differential geometry," Physics Reports 66 no. $6,(1980) 213-393$.

[28] B. P. e. a. Abbott Phys. Rev. X 9 (2019) 031040.

[29] A. del Rio, N. Sanchis-Gual, V. Mewes, I. Agullo, J. A. Font, and J. Navarro-Salas Phys. Rev. Lett. 124 (May, 2020) 211301.

[30] R. M. Wald, General relativity. Chicago Univ. Press, Chicago, IL, 1984.

[31] R. Geroch in Proceedings of a Symposium on Asymptotic Structure of Space-Time, F. Esposito and L. Witten, eds., pp. 1-105. University of Cincinnati, Ohio, 1977.

[32] A. Ashtekar in Surveys in Differential Geometry, L. Bieri and S. T.-Yau, eds., p. 99. International press, Boston, 2015.

[33] A. Ashtekar, Asymptotic quantization: based on 1984 Naples lectures. 1987.

[34] E. Newman and R. Penrose J. Math. Phys. 3 no. 3, (1962) 566-578.

[35] S. Chandrasekhar, The mathematical theory of black holes. Oxford classic texts in the physical sciences. Oxford Univ. Press, Oxford, 2002.

[36] J. Stewart, Advanced General Relativity. Cambridge Monographs on Mathematical Physics. Cambridge University Press, 1991.

[37] H. Bondi, M. G. J. Van der Burg, and A. W. K. Metzner Proc. R. Soc. Lond. A 269 no. 1336, (1962) $21-52$.

[38] F. Alessio and G. Esposito Int. J. Geom. Methods Mod. Phys. 15 no. 02, (2018) 1830002.

[39] A. Ashtekar and B. Bonga Class. Quant. Grav. 34 no. 20, (2017) 20LT01.

[40] A. Ashtekar and B. Bonga Gen. Rel. Grav. 49 no. 9, (2017) 122.

[41] D. J. Griffiths, Introduction to electrodynamics; 4th ed. Pearson, Boston, MA, 2013.

[42] M. A. Berger Plasma Phys. Control. Fusion 41 no. 12B, (1999) B167-B175.

[43] M. Hannam Gen. Rel. Grav. 46 no. 9, (2014).

[44] A. Ashtekar and R. O. Hansen J. Math. Phys. 19 (1978) 1542-1566.

[45] A. Ashtekar and B. C. Xanthopoulos J. Math. Phys. 19 no. 10, (1978) 2216-2222.

[46] E. T. Newman and T. W. J. Unti J. Math. Phys. 3 no. 5, (1962) 891-901. 